\title{
SARS-CoV-2 aberrantly elevates mitochondrial bioenergetics to induce robust virus propagation
}

Hye Jin Shin

Korea Research Institute of Chemical Technology

Keun Bon Ku

Korea Research Institute of Chemical Technology

Gun Young Yoon

Korea Research Institute of Chemical Technology

Hyun-Woo Moon

Korea Research Institute of Chemical Technology

Chonsaeng Kim

Korea Research Institute of Chemical Technology

Bum-Tae Kim

Korea Research Institute of Chemical Technology

Jong-Won Oh

Yonsei University

Aleem Siddiqui

UC San Diego

Seong-Jun Kim ( $\square$ sekim@krict.re.kr)

Korea Research Institute of Chemical Technology https://orcid.org/0000-0001-8504-2872

Article

Keywords:

Posted Date: June 7th, 2021

DOI: https://doi.org/10.21203/rs.3.rs-593889/v1

License: (c) (1) This work is licensed under a Creative Commons Attribution 4.0 International License.

Read Full License 


\section{SARS-CoV-2 aberrantly elevates mitochondrial bioenergetics to induce robust}

4 Hye Jin Shin ${ }^{1}$, Keun Bon $\mathrm{Ku}^{1}$, Gun Young Yun ${ }^{1}$, Hyun-Woo Moon ${ }^{1}$, Chonsaeng Kim ${ }^{1}$, Bum-Tae

$7 \quad{ }^{1}$ Center for Convergent Research of Emerging Virus Infection, Korea Research Institute of Chemical

8 Technology, Daejeon 34114, South Korea; ${ }^{2}$ Department of Biotechnology, Yonsei University, Seoul

9 03722, South Korea; ${ }^{3}$ Division of Infectious Diseases, School of Medicine, University of California,

$12 *$ Corresponding author:

13 Seong-Jun Kim, Ph.D.

14 E-mail: sekim@krict.re.kr

16 Keywords: Severe acute respiratory syndrome coronavirus 2 (SARS-CoV-2); robust virus propagation;

17 aberrant mitochondrial bioenergetics; Epidermal growth factor receptor; EGFR inhibitors 


\section{Abstract}

20 Severe acute respiratory syndrome coronavirus 2 (SARS-CoV-2) is a respiratory pathogen leading

21 to serious multi-organ damage. However, little is known about SARS-CoV-2-induced cellular

22 alterations for understanding robust virus propagation yet. Here we report that SARS-CoV-2 aberrantly

23 elevates mitochondrial bioenergetics and activates epidermal growth factor receptor (EGFR)-mediated

24 cell survival signal cascade for sustaining persistence of SARS-CoV-2. We found that SARS-CoV-2

25 causes increase in mitochondrial transmembrane potential by SARS-CoV-2 RNA-nucleocapsid cluster,

26 thereby abnormally promoting mitochondrial biogenesis and oxidative phosphorylation (OXPHOS)

27 process followed by abundant ATP production. SARS-CoV-2 also activated EGFR signal cascade and

28 subsequent mitochondrial EGFR accumulation which contributes to the maintenance of abnormal

29 OXPHOS and viral propagation. Therapeutic options for the treatment of COVID-19 are still

30 inadequate. The FDA-approved EGFR inhibitors caused a remarkable reduction in SARS-CoV-2

31 propagation. Among EGFR inhibitors, vandetanib showing the highest anti-SARS-CoV-2 efficacy

32 exhibited the potent antiviral activity against various SARS-CoV-2 variants including B.1.1.7 (UK

33 variant) and B.1.351 (SA variant) lineages in both in vitro cell culture and in vivo animal experiments

34 using wild-type aged mouse susceptible to SARS-CoV-2 infection, suggesting that EGFR is an

35 attractive host target for combatting COVID-19. Overall, our results suggest that SARS-CoV-2 induces

36 aberrant mitochondrial dynamics and bioenergetics, which significantly contributes to robust SARS-

37 CoV-2 propagation. 
Severe acute respiratory syndrome coronavirus 2 (SARS-CoV-2) is a global threat leading to

41 serious respiratory illness and death since the first case was reported as a fatal pathogen in $2019^{1,2}$.

42 SARS-CoV-2 phylogenetically closely related to SARS-CoV causing an epidemic in $2003^{3,4}$ is an

43 enveloped, single-stranded positive-sense RNA virus belonging to the family Coronaviridae ${ }^{5}$. SARS-

44 CoV-2 enters into the host cells via host factors angiotensin-converting enzyme carboxypetidase

45 (ACE2) and transmembrane serine protease 2 (TMPRSS2) and utilizes own RNA-dependent RNA

46 polymerase $(\mathrm{RdRp})$ for the replication of viral genome in the cytoplasm ${ }^{6-8}$. In particular, when SARS-

47 CoV-2 infects cells by ACE2-mediated endocytosis, SARS-CoV-2 nucleocapsid protein binding to the

48 packaging signals in the virus RNA (SARS-CoV-2 RNA-nucleocapsid cluster) enters into the cells ${ }^{9,10}$

49 unlike other RNA viruses such as hepatitis $\mathrm{C}$ virus (HCV) and Zika virus (ZIKV) and so on ${ }^{29,30 .}$

Viruses manipulate various intracellular events such as endoplasmic reticulum (ER) stress,

51 oxidative stress, and $\mathrm{Ca}^{2+}$ flux between ER and mitochondria that affects viral propagation and

52 inflammation ${ }^{11-14}$. Particularly, virus infection induces mitochondrial $\mathrm{Ca}^{2+}$ overload thereby resulting

53 in collapse of mitochondria membrane potential $\left(\Delta \Psi_{\mathrm{m}}\right)$ which is the key step in modulating

54 mitochondrial dynamics for the maintenance of cellular homeostasis ${ }^{15,16}$. Recent evidences showed

55 that virus infection induces abnormal mitochondrial dynamics and perturbs mitochondrial

56 bioenergetics that are critical for viral propagation and antiviral innate immune response ${ }^{17,18}$.

The regulation of homoeostasis in host cells is important for the replication of coronaviruses ${ }^{19}$.

58 The pro-survival function of epidermal growth factor receptors (EGFR) is involved in many viral

59 infections. The activation of EGFR is not only required for viral entry to the plasma membrane but

60 also for mitochondrial biogenesis and bioenergetics for viral replication ${ }^{20-25}$. Recent reports showed

61 that beyond a significant contribution of EGFR signaling required for sustaining cell survival and

62 proliferation, EGFR migration to subcellular organelles such as ER and mitochondria following EGFR 
63 activation has been reported ${ }^{36-38}$.

64 In this study, we investigated the SARS-CoV-2-induced mitochondrial alteration which affects 65 robust virus propagation. Our results demonstrate that SARS-CoV-2 elevates mitochondrial 66 bioenergetics efficiency to facilitate SARS-CoV-2 propagation. Unlike other RNA viruses such as

$67 \mathrm{HCV}$ and ZIKV, SARS-CoV-2 infection caused increase in $\Delta \Psi_{\mathrm{m}}$ by viral RNA-nucleocapsid cluster 68 leading to the elongated mitochondria. SARS-CoV-2 infection also increased mitochondrial biogenesis 69 and promoted oxidative phosphorylation (OXPHOS) process followed by abundant ATP production. 70 Further, SARS-CoV-2 activated EGFR/Akt-mediated cell survival signaling and subsequently 71 triggered EGFR translocation to the outer membrane of mitochondria (OMM) and its accumulation, 72 which was convincingly proved by confocal microscopy and Western blot analysis using mitochondrial 73 fractions isolated from SARS-CoV2-infected cells and lung tissue of SARS-CoV-2-infected mouse. 74 SARS-CoV-2-induced activation of EGFR signaling and subsequent EGFR accumulation on the OMM 75 was crucial for sustaining SARS-CoV-2-induced abnormal increase in mitochondrial OXPHOS 76 process and SARS-CoV-2 propagation. Treatment of SARS-CoV-2-infected cells with FDA-approved 77 EGFR inhibitors resulted in drastic reduction in SARS-CoV-2 replication and egress. Furthermore, 78 SARS-CoV-2 viral load in the lung tissue of SARS-CoV-2-infected mouse was dramatically 79 suppressed by oral administration of EGFR inhibitor vandetanib, suggesting that EGFR is a promising 80 host target for combatting SARS-CoV-2 propagation. Overall, our results implicate that SARS-CoV81 2-induced mitochondrial alterations are physiologically relevant in maintenance of persistence of 82 SARS-CoV-2 and pathogenesis. In addition, our results also provide novel insights into the antiviral 83 potential of EGFR inhibitors as promising therapeutic agents in the current lack of effective therapies 84 for the treatment of coronavirus disease 19 (COVID-19). 


\section{SARS-CoV-2 aberrantly elevates mitochondrial bioenergetics}

Recently, we and others have shown that SARS-CoV-2 infects the human cells through host factors

90 ACE2 and TMPRSS2 and replicates in various human epithelial cells ${ }^{7,8}$. In order to investigate SARS-

$91 \mathrm{CoV}-2$-induced alteration in intracellular events which affect a rapid and sustained viral propagation,

92 we first analysed the aspects of SARS-CoV-2 infectivity and replication in several human cells

93 including human normal primary bronchial cells as evidenced by confocal microscopy using anti-

94 SARS-CoV-2 nucleocapsid antibody, real-time qRT-PCR using primer sets specific to SARS-CoV-2

95 nucleocapsid gene, and Western blot analysis with antibodies specific to SARS-CoV-2 spike and

96 nucleocapsid antigens (Extended Data Fig. 1, Extended Data Fig. 2a-c, Extended Data Fig. 3b-c).

97 Interestingly, we observed a prominent elevation in intracellular ATP level paralleled by a rapid 98 increase in viral replication after being infected with SARS-CoV-2 compared to uninfected cells (Fig.

99 1a, Extended Data Fig. 1b, Extended Data Fig. 2b, d). To examine whether SARS-CoV-2 promotes 100 increase in intracellular ATP synthesis during viral replication, we measured the mitochondrial 101 membrane potential $(\Delta \Psi \mathrm{m})$ in SARS-CoV-2-infected cells, as mitochondria are dynamic organelles 102 known as central hub for ATP generation by oxidative phosphorylation ${ }^{26,27}$ and the $\Delta \Psi \mathrm{m}$ is a 103 bioenergetics parameter modulating mitochondria respiration and critical for mitochondrial 104 homeostasis $^{28}$. As a result, unlike other RNA viruses such as HCV and ZIKV ${ }^{29,30}$, SARS-CoV-2 105 infection unusually caused increase in $\Delta \Psi \mathrm{m}$ (Fig. 1b). At this point, we wondered how SARS-CoV-2 106 induces $\Delta \Psi \mathrm{m}$ increase. To substantiate SARS-CoV-2-induced increase in $\Delta \Psi \mathrm{m}$, we focused on the 107 early stage of SARS-CoV-2 infection, as most viruses trigger endoplasmic reticulum (ER) stress at the 108 early stage of infection, leading to the release of $\mathrm{Ca}^{2+}$ from the ER lumen and subsequent $\mathrm{Ca}^{2+}$ 109 accumulation in the mitochondrial matrix, thereby resulting in abnormal $\Delta \Psi \mathrm{m}$ and mitochondrial 110 function ${ }^{14,31}$. Unlike other RNA viruses, SARS-CoV-2 ssRNA and nucleocapsid protein together enters 
111 into the cells when SARS-CoV-2 infects host cells by ACE2-mediated endocytosis 9,10 . Hence, we 112 sought to analyse $\Delta \Psi \mathrm{m}$ in the presence of SARS-CoV-2 RNA and nucleocapsid protein. As shown in

113 Fig. 1c, we first analysed that the expression of SARS-CoV-2 nucleocapsid alone does not significantly 114 affect $\Delta \Psi \mathrm{m}$ (Fig. 1c, left graph). Next, we found that the transfection of SARS-CoV-2 RNA in cells 115 causes loss in $\Delta \Psi \mathrm{m}$ (right graph), whereas the transfection of SARS-CoV-2 RNA in cells expressing 116 nucleocapsid protein induces dramatic increases in $\Delta \Psi \mathrm{m}$ (Fig. 1c, right graph). poly(I:C) was used for 117 the control for $\Delta \Psi \mathrm{m} \operatorname{loss}^{32}$. poly(I:C) did not induce $\Delta \Psi \mathrm{m}$ increase in the presence and absence of 118 SARS-CoV-2 nucleocapsid protein, suggesting that the elaborate structure or formation of SARS-CoV1192 ssRNA and nucleocapsid protein might affect $\Delta \Psi \mathrm{m}$ increase. The accompanying schematic diagram 120 represents that the expression of SARS-CoV-2 RNA and nucleocapsid together causes $\Delta \Psi \mathrm{m}$ increase 121 (Fig. 1c).

122 Next, we examined a mitochondrial shape on confocal microscope, as change in $\Delta \Psi \mathrm{m}$ usually 123 causes alteration in mitochondrial morphology and induces abnormal mitochondrial function. As 124 shown in Fig. 1d, we observed that aberrant elongated and swollen mitochondrial shape is sustained 125 in SARS-CoV-2-infected cells. The accompanying graph presents the quantification of mitochondrial 126 length in SARS-CoV-2-infected cells and uninfected cells shown in confocal microscope. Lack of 127 mitofusin (MFN) creates mitochondrial fusion deficiency and causes severe mitochondrial 128 fragmentation ${ }^{33}$. Hence, we examined the stability of MFN in SARS-CoV-2-infected cells. As shown 129 in Fig. 1e, we found that MFN1/2 is increased and sustained in SARS-CoV-2-infected cells, 130 demonstrating that MFN acts as a modulator for sustaining abnormal mitochondrial elongation in 131 SARS-CoV-2-infected cells. We also examined mitochondrial biogenesis through analysis of 132 mitochondrial DNA (mtDNA) level in SARS-CoV-2-infected cells. Analysis of mitochondrial NADH 133 dehydrogenase 2 (ND2) and cytochrome c oxidase 2 (COX2) reveals that the abnormal increase in 134 mitochondrial mass is maintained during SARS-CoV-2 replication (Fig. 1f).

135 Most of the ATP is mainly generated in mitochondria by the oxidative phosphorylation (OXPHOS) 
136 process $^{34}$. Thus, we subsequently examined a change in the expression levels of mitochondrial 137 OXPHOS complex that might be associated with abundant ATP production on SARS-CoV-2-induced 138 abnormal mitochondrial fusion. As a result, we found that the expression levels of mitochondrial 139 OXPHOS complex I, II, III, IV, and V enzymes are entirely increased in SARS-CoV-2-infected cells 140 (Fig. 1g). Further study by RNAseq and subsequent analysis demonstrated that most of the genes 141 involved in OXPHOS process were upregulated in SARS-CoV-2 (Fig. 1h). Taken together, these 142 results indicate that SARS-CoV-2 induces increase in $\triangle \Psi \mathrm{m}$, mitochondrial biogenesis, and OXPHOS 143 process, thereby abnormally promoting ATP production.

SARS-CoV-2 activates EGFR signal cascade and subsequently induces mitochondrial EGFR translocation

Next, we observed the prolonged survival and proliferation of human cells infected with SARS148 CoV-2 without a distinct viral cytopathogenic effect while we analyse SARS-CoV-2 infectivity in 149 various human cells (Fig. 2a-b, Extended Data Fig. 2e-f, Extended Data Fig.3). Thus, we sought to 150 analyse SARS-CoV-2-induced activation in EGFR signal cascade, as contribution of EGFR signaling 151 is critical for sustaining cell survival and increased cell proliferation ${ }^{35,36}$. As shown in Fig. 2c, SARS152 CoV-2 promoted EGFR gene expression at the transcriptional levels at an early time point of SARS153 CoV-2 infection. Subsequent Western blot analysis also revealed that SARS-CoV-2 results in activation 154 of EGFR-mediated signal cascade as evidenced by increase in the expression levels of phosphorylated 155 EGFR and phosphorylated Akt (Fig. 2d). Together, these results indicate that SARS-CoV-2 promotes 156 EGFR-mediated cell survival and proliferation of infected cells.

Beyond a significant contribution of EGFR signal cascade required for sustaining cell survival and proliferation, EGFR migration to subcellular organelles following EGFR activation has been reported $^{36-38}$. Particularly, mitochondrially localized EGFR contributes to cell survival and regulation 
160 of mitochondrial function ${ }^{36}$. Hence, we further examined if SARS-CoV-2 induces EGFR translocation 161 to mitochondria. We isolated the mitochondrial fraction from SARS-CoV-2-infected cells and analysed 162 SARS-CoV-2-induced mitochondrial EGFP translocation by Western blotting with antibodies specific 163 to phospho-EGFR and EGFR, respectively. As shown in Fig. 2e, SARS-CoV-2 elevated mitochondrial 164 translocation of EGFR and its accumulation compared to those in uninfected cells. A quantitative 165 analysis reveals the difference in relative EGFR accumulation on mitochondrial fraction of SARS166 CoV-2-infected cells (Fig. 2e). Further analysis by confocal microscopy also identified EGFR 167 accumulation on mitochondria of SARS-CoV-2-infected cells (Fig. 2f). Here, we note that SARS-CoV1682 nucleocapsid is localized on mitochondria as evidenced by Western blot analysis and confocal 169 microscopy using antibody specific to SARS-CoV-2 nucleocapsid antigen (Fig. 2e-f).

To strengthen this observation in vivo animal model, we first investigated SARS-CoV-2 infectivity 171 using various age groups of wild-type C57BL/6 mouse to establish wild-type mouse model that is 172 proper for studying SARS-CoV-2 pathogenesis, as we considered that wild-type mouse model is more 173 appropriate for studying SARS-CoV-2 pathogenesis than hACE2 transgenic mouse model which 174 induces an extreme infection of SARS-CoV-2 via overexpression of hACE2 gene. As a result, we 175 found that 15-month aged group of wild-type C57BL/6 mouse is susceptible to SARS-CoV-2 infection 176 (Extended Fig. 4). SARS-CoV-2-infected wild-type 15-month aged mice revealed changes in body 177 weight, survival rate, and viral infectivity in the lung tissue which is very similar with those shown in 178 Golden hamster and Syrian hamster (Extended Fig. 4) ${ }^{39,40}$. Subsequently, we isolated mitochondrial 179 fraction from the lung tissue of wild-type 15-month aged mouse infected with SARS-CoV-2 and 180 analysed mitochondrial EGFR accumulation by Western blotting with anti-EGFR antibody. As shown 181 in Fig. 2g, SARS-CoV-2 infection resulted in enhanced mitochondrial EGFR accumulation in the lung 182 tissue compared to those of uninfected wild-type 15-month aged mouse. A quantitative analysis shows 183 the difference in relative EGFR accumulation on mitochondrial fraction of lung tissue of SARS-CoV184 2-infected mouse (Fig. 2e). Taken together, these results indicate that SARS-CoV-2 activates EGFR 
signal cascade followed by mitochondrial EGFR accumulation during viral infection.

EGFR inhibitors reveals the potent antiviral activity against SARS-CoV-2

Next, we wondered if robust SARS-CoV-2 propagation is suppressed by inhibiting of SARS-CoV-

2-activated EGFR signal cascade. Hence, we evaluated antiviral activity of FDA-approved 12 EGFR

190 inhibitors including gefitinib, olmutinib, erlotinib, lapatinib, bosutinib, cabozantinib, icotinib, 191 vandetanib, brigatinib, afatinib, dacomitinib, and neratinib in SARS-CoV-2-infected cells (Fig. 3a). 192 As shown in Fig. 3b, treatment of SARS-CoV-2-infected cells with EGFR inhibitors caused drastic 193 reduction in the level of extracellular SARS-CoV-2 RNA. Western blot analysis also exhibited that 194 EGFR inhibitors result in the reduction in the expression level of SARS-CoV-2 nucleocapsid protein 195 (Fig. 3c). Among EGFR inhibitors we tested, afatinib, dacomitinib, and neratinib induced a striking 196 cytotoxicity in SARS-CoV-2-infected cells (Table 2). Together, these results suggest that FDAapproved EGFR inhibitors can be utilized as an antiviral therapeutic agent against SARS-CoV-2 infection.

EGFR inhibitor restores SARS-CoV-2-induced abnormal mitochondrial bioenergetics

To substantiate the action of EGFR inhibitors on inhibition of SARS-CoV-2 propagation, we further examined the effect of EGFR inhibitors in SARS-CoV-2-induced mitochondrial alteration. The FDA-approved vandetanib revealed the highest antiviral efficacy against SARS-CoV-2 infection among EGFR inhibitors we tested (Fig. 3b, c). Hence, we examined if vandetanib is capable of restoring SARS-CoV-2-induced abnormal OXPHOS process and subsequent increase in ATP synthesis.

We first analysed that vandetanib reduces mitochondrial EGFR accumulation in SARS-CoV-2-infected cells (Fig. 3d). Further, treatment of SARS-CoV-2-infected cells with vandetanib dramatically restored 
208 SARS-CoV-2-induced increase in intracellular ATP level and the expression levels of mitochondrial

209 OXPHOS complex I, II, III, IV, and V enzymes (Fig. 3e, f). In addition, RNAseq and subsequent

210 analysis demonstrated that vandetanib restores SARS-CoV-2-induced abnormal upregulation in most

211 of genes involved in OXPHOS process (Fig. 3g). Taken together, these results indicate that SARS-

212 CoV-2-induced activation of EGFR signal cascade followed by mitochondrial EGFR translocation

213 contributes to SARS-CoV-2-induced abnormal mitochondrial dynamics and bioenergetics supporting

214 robust SARS-CoV-2 propagation.

216 EGFR inhibitor vandetanib exhibits the potent anti-SARS-CoV-2 activity

217 To substantiate the antiviral effect of vandetanib on SARS-CoV-2 propagation, we first analysed 218 cell cytotoxicity of vandetanib. As shown in Fig. 4a, vandetanib did not significantly affect viability 219 of both uninfected and SARS-CoV-2-infected cells. Vandetanib also led to dose-dependent reduction 220 in SARS-CoV-2 nucleocapsid expression as assessed by Western blot analysis (Fig. 4b). To further 221 understand the action of anti-SARS-CoV-2 effect of vandetanib, we analysed the expression level of 222 intracellular SARS-CoV-2 RNA in infected cells under post-treatment or pre-treatment of vandetanib. 223 As shown in Fig. 4c and d, intracellular SARS-CoV-2 RNA level was drastically declined by post224 treatment of vandetanib, but not pre-treatment as evidenced by real-time qRT-PCR data, suggesting 225 that vandetanib affects an intracellular replication process during SARS-CoV-2 propagation.

We further analysed the in vivo anti-SARS-CoV-2 efficacy of vandetanib using wild-type 15month aged mice. Vandetanib is initially developed as an orally available agent ${ }^{41}$. Hence, we orally 228 administrated vandetanib to SARS-CoV-2-infected mice at dose of $25 \mathrm{mg} / \mathrm{kg}$ daily and terminated 229 SARS-CoV-2-infected mice at 4 days post-infection to analyse intracellular SARS-CoV-2 RNA level 230 in lung tissue of mice (Fig. 4e). As a results, we analysed that vandetanib strongly suppresses SARS231 CoV-2 propagation as assessed by qRT-PCR analysis (Fig. 4f). The intramuscular administration of 
vandetanib in SARS-CoV-2-infected mice did not reveal anti-SARS-CoV-2 efficacy (Fig. 4f).

A combination regimen targeting both virus and host might be more effective for curing COVID-

234 19. Thus, we additionally tested the anti-SARS-CoV-2 effect of vandetanib in combination with 235 remdesivir at a 100-fold lower concentration than the $\mathrm{IC}_{50}$. The advantage of this regimen with EGFR 236 inhibitors in combination with remdesivir is the drastic reduction in toxicity caused by the continuous 237 administration of remdesivir to patients with COVID-19 $9^{42,43}$. As shown in Fig. 4g, treatment of 238 remdesivir in combination with vandetanib caused an additive reduction in intracellular SARS-CoV-2 239 RNA level, suggesting that vandetanib can be utilized in combination with remdesivir without toxicity.

240 Recently, SARS-CoV-2 variants has been constantly spreading worldwide. Thus, we examined if 241 vandetanib reveals antiviral activity against various SARS-CoV-2 (S, V, G, GH, and GR clades) and 242 its variants including B.1.1.7 (United Kingdom variant) and B.1.351 (South Africa variant) lineages. 243 The anti-SARS-CoV-2 activity of vandetanib was evaluated by qRT-PCR analysis and SARS-CoV-2 244 foci reduction assay. As shown in Fig. 5a, treatment of SARS-CoV-2 variants-infected cells with 245 vandetanib caused drastic reduction in the expression levels of extracellular SARS-CoV-2 RNA. 246 Furthermore, vandetanib potently inhibited the infectivity of various SARS-CoV-2 strains in a dose247 dependent manner (Fig. 5b). SARS-CoV-2 strains-induced foci formation was completely inhibited at $24810 \mu \mathrm{M}$ of vandetanib (Fig. 5b). Taken together, these results suggested that vandetanib is a very 249 efficient inhibitor against various SARS-CoV-2 strains.

\section{Discussion}

Although the current COVID-19 pandemic caused by SARS-CoV-2 infection is a severe threat 253 globally, our understanding of persistent SARS-CoV-2 infection and the pathogenesis of COVID-19 254 is still insufficient. In this study, we show that SARS-CoV-2 alters intracellular events and abnormally 255 modulates mitochondrial bioenergetics for robust virus propagation. We found that SARS-CoV-2 
256 increases $\Delta \Psi \mathrm{m}$ which leads to the mitochondrial elongation and swelling. SARS-CoV-2 RNA and 257 nucleocapsid complex promoted $\Delta \Psi \mathrm{m}$ increase. SARS-CoV-2 infection promoted mitochondrial 258 biogenesis and activated mitochondrial OXPHOS process to generate abundant ATP. We also found 259 that SARS-CoV-2 induces EGFR-mediated cell survival signal cascade and subsequently promotes 260 mitochondrial EGFR accumulation which contributes to maintenance of abnormal mitochondrial 261 bioenergetics. Overall, our results suggest that the SARS-CoV-2-modulated abnormal mitochondrial 262 bioenergetics is physiologically relevant to the maintenance of cellular homoeostasis of SARS-CoV263 2-infected cells and robust SARS-CoV-2 replication and propagation (Fig. 6).

The involvement of EGFR internalization and transport to cellular organelles provides a favourable environment for virus replication ${ }^{20-24,44}$. We showed that SARS-CoV-2 stimulates EGFR-mediated cell survival signal and mitochondrial EGFR accumulation for the maintenance of cell proliferation and homeostasis. SARS-CoV-2 infection dramatically triggered the translocation of EGFR to mitochondria as demonstrated by analyses of subcellular fractions and confocal microscpe. Subsequently, SARS269 CoV-2 maintained the abnormally elevated mitochondrial bioenergetics via EGFR internalization and 270 accumulation on mitochondria, which affects viral replication and ATP production. In hepatitis C virus 271 (HCV), EGFR acts as a co-factor for entry and upregulates its internalization and transport to the 272 cellular organelles, and similar processes have been also reported for hepatitis B virus (HBV) and 273 gastroenteritis virus ${ }^{20-25,45}$. SARS-CoV infection also involved the overactivation of EGFR, which 274 leads to increased pulmonary fibrosis ${ }^{46}$. Of note, many respiratory viruses induce EGFR activation in 275 the airway epithelium ${ }^{24,47}$. Moreover, the aberrant expression and activation of EGFR have been 276 implicated in multiple diseases including cancer, diabetes, pulmonary fibrosis, and various lung, heart, 277 and renal diseases ${ }^{35,48-51}$.

Notably, vandetanib caused a remarkable inhibition of SARS-CoV-2 propagation in our study against all clades of SARS-CoV-2. Vandetanib, an FDA-approved EGFR inhibitor, is an inexpensive drug with low toxicity that has been used for the treatment of certain tumours of the thyroid gland ${ }^{52,53}$. 
281 Several EGFR inhibitors have already been shown to block multiple steps of replication in influenza 282 virus $^{54}$ and dengue virus ${ }^{55}$. Therapeutic options in response to the various SARS-CoV2 strains are still 283 limited. In the context of the current COVID-19 pandemic, our study suggests that EGFR inhibitors 284 are a druggable regulator to reduce viral replication by modulation of mitochondrial bioenergetics and 285 EGFR signal cascade against SARS-CoV-2 variants. 


\section{Cell culture}

Human embryonic kidney 293 cells (ATCC), human hepatoma Huh7 cells (JCRB), and human 290 lung epithelial A549 cells (ATCC) were grown in high-glucose DMEM (Gibco) containing 1\% 291 nonessential amino acids (Gibco), 1\% sodium pyruvate (Gibco), and 10\% foetal bovine serum 292 (Hyclone). Human normal primary bronchial cells (ATCC) were grown in bronchial epithelial basal 293 medium (LONZA). All cell lines were incubated in a $5 \% \mathrm{CO}_{2}$ humidified atmosphere at $37^{\circ} \mathrm{C}$.

Viruses

SARS-CoV-2 variants were kindly provided by the Korean Centers for Disease Control and 297 Prevention. SARS-CoV-2 variants used in this study were listed in Table $1^{56}$. SARS-CoV-2 was 298 propagated and prepared in African green monkey kidney Vero E6 cells (ATCC). SARS-CoV-2 299 infections was performed at a multiplicity of infection (MOI) of 1 in biosafety level 3 (BSL3) facility. 300 All experiments using SARS-CoV-2 was handled in the enhanced BSL3 containment laboratory as 301 approved by the Korean Center for Disease Control and Prevention.

303 Reagents and antibodies

The US Food and Drug Administration (FDA)-approved EGFR inhibitors including gefitinib, 305 olmutinib, erlotinib, lapatinib, bosutinib, cabozantinib, icotinib, vandetanib, brigatinib, afatinib, 306 dacomitinib, and neratinib as well as remdesivir were provided by the Korean Chemical Bank at the 307 Korean Research Institute of Chemical Technology (KRICT, Daejeon, South Korea). Primary 308 antibodies used in this study include the following: rabbit monoclonal anti-phospho-EGFR (Y1068, 309 Y1092) (Cell Signaling), rabbit polyclonal anti-EGFR (Millipore), rabbit monoclonal anti-phospho- 
310 Akt (T308) (Cell Signaling), rabbit monoclonal anti-Akt (Cell Signaling), mouse monoclonal anti311 MFN1/2 (Abcam), goat polyclonal anti-VDAC (Abcam), mouse monoclonal anti- $\beta$-actin (Cell

312 Signaling), mouse monoclonal anti-TOM20 (BD), rabbit monoclonal anti-GAPDH (Cell Signaling), 313 mouse monoclonal anti-Hu total OxPhos complex (Thermo Fisher Scitific), rabbit monoclonal anti-

314 SARS-CoV-2 nucleoprotein (Sino Biological), and rabbit polyclonal anti-SARS-CoV-2 spike (Sino 315 Biological). The secondary antibodies used for immunofluorescence were Alexa Fluor 633 goat anti316 rabbit antibody. The secondary antibodies used for western blot analyses were HRP-conjugated anti317 mouse IgG (Invitrogen, Waltham, MA, USA) and HRP-conjugated anti-rabbit IgG (Invitrogen).

\section{Western blot analysis}

320 For the immunoblotting assay, denatured protein samples were prepared by boiling in SDS loading 321 buffer (50 mM Tris, pH 6.8, 2\% SDS, 10\% glycerol, 0.1 M DTT, 0.05\% bromophenol blue). Proteins 322 were separated via SDS-PAGE and transferred onto PVDF membranes (Millipore). The membranes 323 were blocked with 5\% skim milk in PBS containing 0.1\% Tween-20 (PBS-T) and stained with 324 antibodies against the indicated proteins. The membranes were washed three times with PBS-T and 325 then developed using the western ECL Femto Kit (LPS Solution). Images were captured using 326 ImageQuant LAS 4000 (GE Healthcare). The intensity of protein expression was quantified using 327 ImageJ (Bethesda).

\section{TMRE (tetramethylrhodamine, ethyl ester) assay}

A Mitochondrial Membrane Potential Assay kit (BioVision) was used according to the manufacturer's instructions. A total cells were incubated with the fluorescent TMRE (200nM) dye for $33220 \mathrm{~min}$ at $37^{\circ} \mathrm{C}, 5 \% \mathrm{CO} 2$. For a negative control, FCCP (carbonyl cyanide 4-(trifluoromethoxy) 
334 addition. Following incubation, the stained cells were harvested and gently washed with assay buffer.

335 The fluorescence signal was measured at 549/575 $\mathrm{nm}$ of excitation/emission wavelengths using a 336 Synergy H1 multi-mode microplate reader (BioTek). Two technical and biological replicates were 337 performed for each sample.

\section{Real-time qRT-PCR and qPCR}

340 To analyse the expression levels of EGFR and SARS-CoV-2 viral genes, total RNA was extracted 341 from cells using the RNeasy Mini Kit (Qiagen, Hilden, Germany). Extracellular viral RNA from 342 supernatants was isolated using a QIAamp Viral RNA Mini Kit (Qiagen). The cellular RNA level of 343 EGFR was quantified by real-time qRT-PCR using the One-Step SyBr Green RT-PCR Kit II (Takara). 344 The intracellular and extracellular SARS-CoV-2 RNA copy numbers were determined by real-time 345 qRT-PCR using the One Step PrimeScript III RT-PCR Kit (Takara). The following primer sets were 346 used for RT-PCR: EGFR forward, 5'-AGGCAGGAGTAACAAGCTCAC; EGFR reverse, 5'347 ATGAGGACATAACAAGCCACC; SARS-CoV-2-N forward, 5'-TTACAAACATTG GCCGCAAA; 348 SARS-CoV-2-N reverse 5'-GCGCGACATTCCGAAGAA; SARS-CoV-2-N probe 5'-FAM349 ACAATTTGCCCCCAGCGCTTCAG-BHQ1; $\beta$-actin forward, 5'-ACAGAGCCTCGCCTTTG; $\beta$ 350 actin $\quad$ reverse, $\quad$ '-CCTTGCACATGCCGGAG, $\quad \beta$-actin $\quad$ probe; $\quad 5$ '-/56351 FAM/TCATCCATG/ZEN/GTGAGCTGGCGG/3IABkFQ.

352 To analyze the expression levels of mitochondrial DNA, total cellular DNA was extracted from 353 the cells using AllPrep DNA kit (Qiagen) and subsequently quantified by qPCR using DyNAmo HS 354 SYBR Green qPCR kit according to the manufacturer's instructions. The following primer sets were 355 used for qPCR: ND-2 forward, 5'-TAGCCCCCTTTCACTTCTGA; ND-2 reverse, 5'356 GCGTAGCTGGGTTTGGTTTA; COX-2 forward, 5'-GGCCACCAATGGTACTGAAC; COX-2 
reverse, 5'-CGGGAATTGCATCTGTTTTT. Real-time qPCR was conducted using the QuantStudio3

358 Real-Time PCR System (Applied Biosystems).

\section{Immunofluorescence}

361 For immunofluorescence, cells grown on glass cover slips were infected with SARS-CoV-2 at an 362 MOI of 1 . At 2 days post-infection, cells were fixed with 4\% paraformaldehyde and permeabilized 363 with $0.2 \%$ Triton X-100 in phosphate-buffered saline. Cells were blocked in blocking buffer (2\% BSA, 364 PBS) and immunostained with the indicated antibodies. The coverslips were mounted in antifade 365 medium containing DAPI (Vector Laboratories). Images were captured using a Zeiss LMS700 366 confocal laser-scanning microscope and evaluated using ZEM 2012 SP5.

Cell viability and ATP levels were measured using a Synergy H1 Microplate Reader (BioTek) using the CellTiter-Glo assay (Promega) according to the manufacturer's instructions. Total cell number and cell viability were quantified using a haemocytometer and the Trypan Blue exclusion method.

\section{Subcellular fractionation}

374 Mitochondria were isolated from SARS-CoV-2-infected cells or lung tissue of SARS-CoV-2375 infected mice using a Mitochondria Isolation Kit (Thermo Scientific) according to the manufacturer's 376 instructions. Protein extracted from each cellular fraction was analysed by western blotting with the 377 indicated antibodies. 
Mouse experiments were approved by the Institutional Animal Care and Use Committee (IACUC)

381 of the Korea Research Institute of Chemical Technology (2020-8B-07-01). Nine wild-type C57BL/6

382 aged mice (15 month old) were evenly divided into three groups and housed in the ABSL-3 facility.

383 Mice from each group were lightly euthanized with isoflurane and injected with SCoV2 $\left(1 \times 10^{5}\right.$

$384 \mathrm{pfu} /$ head, clade S) into each mouse via the intranasal route. Mice were orally administered vehicle or 385 vandetanib (25 mg/kg per mouse) once daily. All mice was terminated for further analysis at 4 day 386 post-infection. Mouse lung samples were washed with PBS and harvested to test the inhibition of 387 SCoV2. Tissue samples from the lung were homogenized in cold phosphate-buffered saline (PBS) 388 solution with a FastPrep-24 homogenizer (MP Biomedicals) for 5 cycles $(20 \mathrm{~s} \mathrm{on} / 20 \mathrm{~s}$ off). Three 389 additional freeze-thaw cycles were performed at $-80^{\circ} \mathrm{C}$, and cell debris was removed by centrifugation 390 at $13000 \mathrm{rpm}$ for $1 \mathrm{~min}$ to measure the viral RNA and proteins in the lung.

\section{FFU (Focus forming unit) assay}

The titer of infectious virus in the culture medium was determined by FFU assay. Vero E6 cells were seeded in a 96 well plate. The infectious cell culture medium was used to infect the cells and, the plates were incubated at $37{ }^{\circ} \mathrm{C}$. After $8 \mathrm{~h}$, cells were washed with PBS and fixed with $4 \%$ 396 paraformaldehyde and permeabilized with methanol. SCoV2 foci were developed using anti-SCoV2 $397 \mathrm{~N}$ antibody followed by addition of horseradish peroxidase-conjugated goat anti-rabbit IgG secondary antibody and TMB substrate (Promega). Plates were incubated in dark condition at room temperature

399 for $30 \mathrm{~min}$. The plates were washed and dried for observation under a light microscope. The number 400 of SCoV2 positive foci was scanned and counted in each well using an ImmunoSpot reader (CTL, 401 Shaker Height, OH). 
Raw reads were mapped to the human genome assembly hg38 using the aligner STAR v.2.4.0b ${ }^{57}$.

405 To measure gene expression level, the gene annotation database of the species was used with Cufflinks $406 \mathrm{v} 2.1 .1^{58}$. For differential expressed gene (DEG) analysis, gene level count data were generated by 407 using HTSeq-count v0.6.1p $1^{59}$. The $\mathrm{R}$ package called $\mathrm{TCC}^{60}$ was used to identify DEGs. For 408 comparing tag count data, this package applies robust normalization strategies. Normalization factors 409 were measured by using the iterative DEGES/edgeR method. For correcting errors caused by multiple410 testing, the DEGs were identified based on the qvalue threshold less than $0.05^{61}$.

\section{Statistical analysis}

413 Unpaired Student's $t$-tests and one-way ANOVA were performed using GraphPad Prism 8 (Graph 414 Pad, La Jolla, CA, USA). A value of $\mathrm{P}<0.05$ was considered significant.

\section{Acknowledgements}

This work was supported by a National Research Council of Science \& Technology (NST) grant 418 through the Korean government (MSIP) (No. CRC-16-01-KRICT) to S.-J.K. We thank all members of 419 the vaccine R\&D team at the Center for Convergent Research for Emerging Virus Infection (CEVI) 420 and Dr. Sunkyung Lee (Korea Research Institute of Chemical Technollgy) for their technical support 421 and in-depth discussions. The Chemical Library used in this study was kindly provided by the Korea 422 Chemical Bank (www.chembank.org) of the Korea Research Institute of Chemical Technology. SARS423 CoV-2 variants were kindly provided by the Korean Centers for Disease Control and Prevention.

\section{Author contributions}

H.J.S. and S.-J.K. conceptualised and designed the study. H.J.S., K.B.K., G.Y.Y., H.-W.M., and 
427 C.K. conducted the experiments. H.J.S., B.-T.K., J.-W.O., A.S., and S.-J.K. analysed the data. H.J.S. 428 and S.-J.K. wrote the paper.

429

\section{$430 \quad$ Competing interests}

431 The authors declare no competing interests. 
Ahn, D. G. et al. Current Status of Epidemiology, Diagnosis, Therapeutics, and Vaccines for Novel Coronavirus Disease 2019 (COVID-19). J Microbiol Biotechnol 30, 313-324, doi:10.4014/jmb.2003.03011 (2020).

2 Zhu, N. et al. A Novel Coronavirus from Patients with Pneumonia in China, 2019. N Engl J Med 382, 727-733, doi:10.1056/NEJMoa2001017 (2020).

3 Marra, M. A. et al. The Genome sequence of the SARS-associated coronavirus. Science 300, 1399-1404, doi:10.1126/science.1085953 (2003).

4 Drosten, C. et al. Identification of a novel coronavirus in patients with severe acute respiratory syndrome. N Engl J Med 348, 1967-1976, doi:10.1056/NEJMoa030747 (2003).

5 Lu, R. et al. Genomic characterisation and epidemiology of 2019 novel coronavirus: implications for virus origins and receptor binding. Lancet 395, 565-574, doi:10.1016/S01406736(20)30251-8 (2020).

6 Hartenian, E. et al. The molecular virology of coronaviruses. J Biol Chem 295, 12910-12934, doi:10.1074/jbc.REV120.013930 (2020).

7 Lee, S., Yoon, G. Y., Myoung, J., Kim, S. J. \& Ahn, D. G. Robust and persistent SARS-CoV-2 infection in the human intestinal brush border expressing cells. Emerg Microbes Infect 9, 21692179, doi:10.1080/22221751.2020.1827985 (2020).

8 Hoffmann, M. et al. SARS-CoV-2 Cell Entry Depends on ACE2 and TMPRSS2 and Is Blocked by a Clinically Proven Protease Inhibitor. Cell 181, 271-280 e278, doi:10.1016/j.cell.2020.02.052 (2020).

$9 \mathrm{Li}, \mathrm{W}$. et al. Angiotensin-converting enzyme 2 is a functional receptor for the SARS coronavirus. Nature 426, 450-454, doi:10.1038/nature02145 (2003).

10 Li, F., Li, W., Farzan, M. \& Harrison, S. C. Structure of SARS coronavirus spike receptorbinding domain complexed with receptor. Science 309, 1864-1868, doi:10.1126/science.1116480 (2005).

11 Piccoli, C. et al. Hepatitis C virus protein expression causes calcium-mediated mitochondrial bioenergetic dysfunction and nitro-oxidative stress. Hepatology 46, 58-65, doi:10.1002/hep.21679 (2007).

12 Tardif, K. D., Waris, G. \& Siddiqui, A. Hepatitis C virus, ER stress, and oxidative stress. Trends Microbiol 13, 159-163, doi:10.1016/j.tim.2005.02.004 (2005).

13 Gong, G., Waris, G., Tanveer, R. \& Siddiqui, A. Human hepatitis C virus NS5A protein alters intracellular calcium levels, induces oxidative stress, and activates STAT-3 and NF-kappa B. Proc Natl Acad Sci U S A 98, 9599-9604, doi:10.1073/pnas.171311298 (2001).

$14 \mathrm{He}, \mathrm{B}$. Viruses, endoplasmic reticulum stress, and interferon responses. Cell Death Differ 13, 393-403, doi:10.1038/sj.cdd.4401833 (2006).

15 Kim, S. J., Ahn, D. G., Syed, G. H. \& Siddiqui, A. The essential role of mitochondrial dynamics in antiviral immunity. Mitochondrion 41, 21-27, doi:10.1016/j.mito.2017.11.007 (2018).

16 Elesela, S. \& Lukacs, N. W. Role of Mitochondria in Viral Infections. Life (Basel) 11, doi:10.3390/life11030232 (2021).

17 Khan, M., Syed, G. H., Kim, S. J. \& Siddiqui, A. Mitochondrial dynamics and viral infections: A close nexus. Biochim Biophys Acta 1853, 2822-2833, doi:10.1016/j.bbamcr.2014.12.040 (2015).

18 Benard, G. \& Rossignol, R. Ultrastructure of the mitochondrion and its bearing on function and bioenergetics. Antioxid Redox Signal 10, 1313-1342, doi:10.1089/ars.2007.2000 (2008).

19 Tan, Y. J., Lim, S. G. \& Hong, W. Regulation of cell death during infection by the severe acute 
respiratory syndrome coronavirus and other coronaviruses. Cell Microbiol 9, 2552-2561, doi:10.1111/j.1462-5822.2007.01034.x (2007).

20 Lupberger, J. et al. EGFR and EphA2 are host factors for hepatitis C virus entry and possible targets for antiviral therapy. Nat Med 17, 589-595, doi:10.1038/nm.2341 (2011).

21 Diao, J. et al. Hepatitis C virus induces epidermal growth factor receptor activation via CD81 binding for viral internalization and entry. $J$ Virol 86, 10935-10949, doi:10.1128/JVI.00750-12 (2012).

22 Iwamoto, M. et al. Epidermal growth factor receptor is a host-entry cofactor triggering hepatitis B virus internalization. Proc Natl Acad Sci $U$ S A 116, 8487-8492, doi:10.1073/pnas.1811064116 (2019).

23 Iwamoto, M. et al. The machinery for endocytosis of epidermal growth factor receptor coordinates the transport of incoming hepatitis B virus to the endosomal network. $J$ Biol Chem 295, 800-807, doi:10.1074/jbc.AC119.010366 (2020).

24 Yang, L. et al. Porcine Epidemic Diarrhea Virus-Induced Epidermal Growth Factor Receptor Activation Impairs the Antiviral Activity of Type I Interferon. $J$ Virol 92, doi:10.1128/JVI.02095-17 (2018).

$25 \mathrm{Hu}, \mathrm{W}$., Zhang, S., Shen, Y. \& Yang, Q. Epidermal growth factor receptor is a co-factor for transmissible gastroenteritis virus entry. Virology 521, 33-43, doi:10.1016/j.virol.2018.05.009 (2018).

26 Chan, D. C. Mitochondria: dynamic organelles in disease, aging, and development. Cell 125, 1241-1252, doi:10.1016/j.cell.2006.06.010 (2006).

27 Hatefi, Y. The mitochondrial electron transport and oxidative phosphorylation system. Annu Rev Biochem 54, 1015-1069, doi:10.1146/annurev.bi.54.070185.005055 (1985).

28 Nicholls, D. G. Mitochondrial membrane potential and aging. Aging Cell 3, 35-40, doi:10.1111/j.1474-9728.2003.00079.x (2004).

29 Ando, T. et al. Visualization and measurement of ATP levels in living cells replicating hepatitis C virus genome RNA. PLoS Pathog 8, e1002561, doi:10.1371/journal.ppat.1002561 (2012).

30 Yang, S. et al. Zika Virus-Induced Neuronal Apoptosis via Increased Mitochondrial Fragmentation. Front Microbiol 11, 598203, doi:10.3389/fmicb.2020.598203 (2020).

31 Deniaud, A. et al. Endoplasmic reticulum stress induces calcium-dependent permeability transition, mitochondrial outer membrane permeabilization and apoptosis. Oncogene 27, 285299, doi:10.1038/sj.onc.1210638 (2008).

32 Harashima, N., Minami, T., Uemura, H. \& Harada, M. Transfection of poly(I:C) can induce reactive oxygen species-triggered apoptosis and interferon-beta-mediated growth arrest in human renal cell carcinoma cells via innate adjuvant receptors and the 2-5A system. Mol Cancer 13, 217, doi:10.1186/1476-4598-13-217 (2014).

33 Chen, H., Chomyn, A. \& Chan, D. C. Disruption of fusion results in mitochondrial heterogeneity and dysfunction. J Biol Chem 280, 26185-26192, doi:10.1074/jbc.M503062200 (2005).

34 Mookerjee, S. A., Gerencser, A. A., Nicholls, D. G. \& Brand, M. D. Quantifying intracellular rates of glycolytic and oxidative ATP production and consumption using extracellular flux measurements. J Biol Chem 292, 7189-7207, doi:10.1074/jbc.M116.774471 (2017).

35 Wee, P. \& Wang, Z. Epidermal Growth Factor Receptor Cell Proliferation Signaling Pathways. Cancers (Basel) 9, doi:10.3390/cancers9050052 (2017).

36 Yue, X. et al. Mitochondrially localized EGFR is subjected to autophagic regulation and implicated in cell survival. Autophagy 4, 641-649, doi:10.4161/auto.5971 (2008).

37 Cao, X., Zhu, H., Ali-Osman, F. \& Lo, H. W. EGFR and EGFRvIII undergo stress- and EGFR kinase inhibitor-induced mitochondrial translocalization: a potential mechanism of EGFRdriven antagonism of apoptosis. Mol Cancer 10, 26, doi:10.1186/1476-4598-10-26 (2011).

38 Tan, X., Lambert, P. F., Rapraeger, A. C. \& Anderson, R. A. Stress-Induced EGFR Trafficking: 
Mechanisms, Functions, and Therapeutic Implications. Trends Cell Biol 26, 352-366, doi:10.1016/j.tcb.2015.12.006 (2016).

39 Sia, S. F. et al. Pathogenesis and transmission of SARS-CoV-2 in golden hamsters. Nature 583, 834-838, doi:10.1038/s41586-020-2342-5 (2020).

40 Imai, M. et al. Syrian hamsters as a small animal model for SARS-CoV-2 infection and countermeasure development. Proc Natl Acad Sci $U$ S A 117, 16587-16595, doi:10.1073/pnas.2009799117 (2020).

41 Herbst, R. S., Heymach, J. V., O'Reilly, M. S., Onn, A. \& Ryan, A. J. Vandetanib (ZD6474): an orally available receptor tyrosine kinase inhibitor that selectively targets pathways critical for tumor growth and angiogenesis. Expert Opin Investig Drugs 16, 239-249, doi:10.1517/13543784.16.2.239 (2007).

42 Goldman, J. D. et al. Remdesivir for 5 or 10 Days in Patients with Severe Covid-19. N Engl J Med, doi:10.1056/NEJMoa2015301 (2020).

43 Wang, Y. et al. Remdesivir in adults with severe COVID-19: a randomised, double-blind, placebo-controlled, multicentre trial. Lancet 395, 1569-1578, doi:10.1016/S01406736(20)31022-9 (2020).

44 Chu, H. et al. Comparative tropism, replication kinetics, and cell damage profiling of SARSCoV-2 and SARS-CoV with implications for clinical manifestations, transmissibility, and laboratory studies of COVID-19: an observational study. Lancet Microbe 1, e14-e23, doi:10.1016/S2666-5247(20)30004-5 (2020).

45 Hondermarck, H., Bartlett, N. W. \& Nurcombe, V. The role of growth factor receptors in viral infections: An opportunity for drug repurposing against emerging viral diseases such as COVID-19? FASEB Bioadv 2, 296-303, doi:10.1096/fba.2020-00015 (2020).

46 Venkataraman, T., Coleman, C. M. \& Frieman, M. B. Overactive Epidermal Growth Factor Receptor Signaling Leads to Increased Fibrosis after Severe Acute Respiratory Syndrome Coronavirus Infection. J Virol 91, doi:10.1128/JVI.00182-17 (2017).

47 Ueki, I. F. et al. Respiratory virus-induced EGFR activation suppresses IRF1-dependent interferon lambda and antiviral defense in airway epithelium. $J$ Exp Med 210, 1929-1936, doi:10.1084/jem.20121401 (2013).

48 Chen, J. et al. Expression and Function of the Epidermal Growth Factor Receptor in Physiology and Disease. Physiol Rev 96, 1025-1069, doi:10.1152/physrev.00030.2015 (2016).

$49 \mathrm{Li}, \mathrm{Z}$. et al. Inhibition of Epidermal Growth Factor Receptor Activation Is Associated With Improved Diabetic Nephropathy and Insulin Resistance in Type 2 Diabetes. Diabetes 67, $1847-$ 1857, doi:10.2337/db17-1513 (2018).

50 Rayego-Mateos, S. et al. Role of Epidermal Growth Factor Receptor (EGFR) and Its Ligands in Kidney Inflammation and Damage. Mediators Inflamm 2018, 8739473, doi:10.1155/2018/8739473 (2018).

51 Venkataraman, T. \& Frieman, M. B. The role of epidermal growth factor receptor (EGFR) signaling in SARS coronavirus-induced pulmonary fibrosis. Antiviral Res 143, 142-150, doi:10.1016/j.antiviral.2017.03.022 (2017).

52 Fallahi, P. et al. The safety and efficacy of vandetanib in the treatment of progressive medullary thyroid cancer. Expert Rev Anticancer Ther 16, 1109-1118, doi:10.1080/14737140.2016.1238764 (2016).

53 Tsang, V. H., Robinson, B. G. \& Learoyd, D. L. The safety of vandetanib for the treatment of thyroid cancer. Expert Opin Drug Saf 15, 1107-1113, doi:10.1080/14740338.2016.1201060 (2016).

54 Kumar, N., Liang, Y., Parslow, T. G. \& Liang, Y. Receptor tyrosine kinase inhibitors block multiple steps of influenza a virus replication. $J$ Virol 85, 2818-2827, doi:10.1128/JVI.0196910 (2011).

55 Duran, A., Valero, N., Mosquera, J., Fuenmayor, E. \& Alvarez-Mon, M. Gefitinib and 
pyrrolidine dithiocarbamate decrease viral replication and cytokine production in dengue virus infected human monocyte cultures. Life Sci 191, 180-185, doi:10.1016/j.lfs.2017.10.027 (2017).

56 Kim, J. M. et al. Identification of Coronavirus Isolated from a Patient in Korea with COVID19. Osong Public Health Res Perspect 11, 3-7, doi:10.24171/j.phrp.2020.11.1.02 (2020).

57 Dobin, A. et al. STAR: ultrafast universal RNA-seq aligner. Bioinformatics 29, 15-21, doi:10.1093/bioinformatics/bts635 (2013).

58 Trapnell, C. et al. Transcript assembly and quantification by RNA-Seq reveals unannotated transcripts and isoform switching during cell differentiation. Nat Biotechnol 28, 511-515, doi:10.1038/nbt.1621 (2010).

59 Anders, S., Pyl, P. T. \& Huber, W. HTSeq--a Python framework to work with high-throughput sequencing data. Bioinformatics 31, 166-169, doi:10.1093/bioinformatics/btu638 (2015).

60 Sun, J., Nishiyama, T., Shimizu, K. \& Kadota, K. TCC: an R package for comparing tag count data with robust normalization strategies. BMC Bioinformatics 14, 219, doi:10.1186/14712105-14-219 (2013).

61 Yoav Benjamini, Y. H. Controlling the False Discovery Rate: A Practical and Powerful Approach to Multiple Testing. Journal of the Royal Statistical Society: Series B (Methodological) 57, 289-300, doi:10.1111/j.2517-6161.1995.tb02031.x (1995). 
601 Fig. 1 | SCoV2 induces aberrant mitochondrial elongation and alterations in mitochondrial 602 bioenergetics. a, Quantification of the elevated level of intracellular ATP in SCoV2-infected HEK293 603 cells at the indicated time points. Data, average of two independent experiments (mean $\pm \mathrm{SD} ; \mathrm{n}=3$; $604 * \mathrm{p}<0.001) . \mathbf{b}$, Quantification of $\Delta \Psi_{\mathrm{m}}$ increase in HEK293 cells infected with SCoV2 at an MOI of 1. $605 \Delta \Psi_{\mathrm{m}}$ in SCoV2-infected cells were monitored by TMRE assay at 1 day post-infection (mean $\pm \mathrm{SD}$; $\mathrm{n}=3 ; * * \mathrm{p}<0.005) . \Delta \Psi_{\mathrm{m}}$, mitochondrial membrane potential; FCCP, synthetic mitochondrial uncoupler, a control for $\Delta \Psi_{\mathrm{m}}$ loss; accompanying diagram represents SCoV2-induced increase in $\Delta \Psi_{\mathrm{m}}$. c, 608 Quantification of $\Delta \Psi_{\mathrm{m}}$ increase by SCoV2 RNA-N cluster. HEK293 cells transiently expressing $609 \mathrm{SCoV} 2 \mathrm{~N}$ gene were transfected with $\operatorname{SCoV} 2 \mathrm{RNA}(1 \mu \mathrm{g} / \mathrm{ml}) . \Delta \Psi_{\mathrm{m}}$ in cells were monitored by TMRE 610 assay at $4 \mathrm{~h}$ post-transfection (mean $\pm \mathrm{SD} ; \mathrm{n}=3 ;{ }^{*} \mathrm{p}<0.001, * * * \mathrm{p}<0.01$ ). poly(I:C), synthetic analog of 611 double-stranded RNA, a control for $\Delta \Psi_{\mathrm{m}}$ loss; accompanying diagram represents SCoV2 RNA-N 612 cluster-induced increase in $\Delta \Psi_{\mathrm{m}}$. d, Confocal microscopy showing the elongated and swollen shape of 613 mitochondria in SCoV2-infected cells. SCoV2-infected HEK293 cells were stained with SCoV2-S 614 (red) and TOM20 (green) antibodies. Nuclei, DAPI (blue); Infection, SCoV2 spike (S) antigen; 615 accompanying graph represents the quantification of mitochondrial length by MBF ImageJ. e, Western blot analysis of MFN1 expression in SCoV2-infected cells. At 1 days post-infection, mitochondrial 617 fraction (Mito) isolated from SCoV2-infected HEK293 cells was analyzed by immunoblotting with 618 anti-MFN1 antibody. f, qPCR data showing the increase of mitochondrial DNA in SCoV2-infected 619 cells. At 1 day post-infection, the expression level of mitochondrial ND2 and COX2 DNA was 620 analyzed by real-time qPCR. GAPDH was used to normalized changes in ND2 and COX2 expression 621 (mean $\pm \mathrm{SD} ; \mathrm{n}=3 ;{ }^{*} \mathrm{p}<0.001$ ). $\mathbf{g}$, Western blot analysis of mitochondrial respiratory chain complex 622 enzyme expression in SCoV2-infected cells. At a day post-infection, the expression level of complex 623 I, II, III, IV, and V enzymes was analyzed by immunoblotting with anti-Hu total OXPHOS complex 624 antibody. $\beta$-actin, an internal loading control; accompanying graph represents the quantification of 
625 increase in complex I, II, III, IV, and V expression in SCoV2-infected cells by ImageJ. P value was

626 calculated by using an unpaired Student's t-test. $\mathbf{h}$, Heat maps of relative mRNA and protein expression

627 of the indicated mitochondrial OXPHOS genes isolated from uninfected and SARS-CoV-2-infected

628 cells. Each box indicates an average of three independent experiments. Colour indicates $\log 1$ fold-

629 change for uninfected vs SARS-CoV-2-infected cells.

Fig. 2 | SCoV2 promotes EGFR-mediated cell survival signal cascade and mitochondrial EGFR

632 translocation. a, Quantification of SCoV2-infected HEK293 cell viability. Total number of viable 633 HEK293 cells infected with SCoV2 at an MOI of 1 was measured at the indicated time points. b, Light 634 microscopic analysis of SCoV2-infected HEK293 cells at 5 days post-infection. c, Quantitative 635 analysis of EGFR gene expression in SCoV2-infected cells. At 1 day post-infection, the expression 636 level of EGFR mRNA in SCoV2-infected cells were analysed by real-time qRT-PCR ( $m e a n \pm S D ; n=3$; $637 * \mathrm{p}<0.001)$. $\mathbf{d}$, Western blot analysis of EGFR-mediated signal cascade in SCoV2-infected cells. At 1 638 days post-infection, whole cell lysates of SCoV2-infected HEK293 cells were analysed by 639 immunoblotting with antibodies specific to p-EGFR, EGFR, p-Akt (Thr308), and Akt. $\beta$-actin, an 640 internal loading control. e, SCoV2-induced mitochondrial accumulation of EGFR. Cytosolic and 641 mitochondrial fractions isolated from uninfected and SCoV2-infected HEK293 cells were evaluated 642 by immunoblotting with antibodies specific to p-EGFR and EGFR. Fractions: purified cytoplasm, Cyto; 643 purified mitochondria, Mito. Organelle marker: TOM20, mitochondria. Infection marker: SCoV2 644 nucleocapsid $(\mathrm{N})$ antigen. The relative intensity of EGFR normalized to TOM20 and p-EGFR 645 normalized to EGFR respectively was analysed by ImageJ. f, Confocal microscopy showing the 646 mitochondrial accumulation of EGFR in SCoV2-infected cells. SCoV2-infected HEK293 cells were 647 stained with EGFR (green), VDAC (red), and SCoV2-N (white on upper panel, blue on lower panel) 648 antibodies. Infection, SCoV2 nucleocapsid (N) antigen; Arrow (white), colocalization of VDAC and 649 SCoV2 N antigen. g, Western blot analysis of mitochondrial EGFR accumulation in lung tissue of 
650 wild-type aged mouse infected with SCoV2. Mitochondrial fraction (Mito) isolated from lung tissue 651 of uninfected and SCoV2-infected wild-type aged mouse were analysed by immunoblotting with

652 EGFR antibody. Organelle marker: TOM20, mitochondria. Infection marker: SCoV2 nucleocapsid (N) 653 antigen. The relative intensity of EGFR expression level normalized to TOM20 was analysed by 654 ImageJ.

655

Fig 3 | Tyrosine kinase inhibitors targeting EGFR signaling pathway suppresses SCoV2

propagation. a, A scheme for screening antiviral effect of EGFR inhibitors against SCoV2 propagation. At 4 h post-infection, HEK293 cells infected with SCoV2 at an MOI of 1 were washed with fresh cell culture media 5 times and subsequently treated with the indicated EGFR inhibitors (10 $\mu \mathrm{M})$ for $44 \mathrm{~h}$. Cell culture supernatant and pellet were used for further analysis. b, Extracellular RNA isolated from culture supernatant of SCoV2-infected cells was used for the analysis of SCoV2 RNA level by qRT-PCR using PCR primers set specific to the SCoV2 N gene. c, Whole cell lysates of 663 SCoV2-infected cells were analysed with SCoV2 N antibody. Accompanying graph, ImageJ quantification of SCoV2 N expression level. d, Rescue of SCoV2-induced abnormal mitochondrial accumulation of EGFR. Cytosolic and mitochondrial fractions isolated from uninfected and SCoV2infected cells treated with vandetanib were analysed by immunoblotting with EGFR antibody.

667 Fractions: purified cytoplasm, Cyto; purified mitochondria, Mito. Organelle marker: TOM20, 668 mitochondria; GAPDH, cytoplasm. SCoV2 infection marker: SCoV2 nucleocapsid (N). The relative 669 intensity of EGFR normalized to TOM20 was analysed by ImageJ. e, Rescue of SCoV2-induced 670 elevation in intracellular ATP level by vandetanib treatment. At 1 day post-treatment of vandetanib, 671 intracellular ATP level in SCoV2-infected HEK293 cells was analysed as described in the Materials 672 and Methods. Data, average of two independent experiments (mean $\pm \mathrm{SD} ; \mathrm{n}=3 ;{ }^{*} \mathrm{p}<0.001$ ). f, Western 673 blot analysis of mitochondrial respiratory chain complex enzyme expression. At 1 day post-treatment 674 of vandetanib, the expression level of complex I, II, III, IV, and V enzymes was analysed by 
immunoblotting with anti-Hu total OxPhos complex antibody. $\beta$-actin, an internal loading control;

676 SCoV2 N, infection marker. $\mathbf{g}$, Heat maps of relative mRNA and protein expression of the indicated

677 mitochondrial OXPHOS genes isolated from SARS-CoV-2-infected and vandetanib-treated SARS-

678 CoV-2-infected cells. Each box indicates an average of three independent experiments. Colour 679 indicates $\log 1$ fold-change for SARS-CoV-2-infected vs vandetanib-treated SARS-CoV-2-infected 680 cells.

681

682 Fig 4 | Vandetanib is a potent antiviral agent for SCoV2 propagation. a, Viability of SCoV2683 infected cells treated with vandetanib for 2 days. Cell viability was analysed as described in the 684 Materials and Methods. b, Dose dependent antiviral effect of vandetanib against SCoV2 infection. At 2 days post-treatment, whole cell lysates of SCoV2-infected cells treated with vandetanib at the 686 indicated concentrations were analysed by immunoblotting with SCoV2 $\mathrm{N}$ antibody. The relative 687 intensity of SCoV2 N expression level normalized to $\beta$-actin was analysed by ImageJ. $\mathbf{c}$, Anti-SCoV2 688 effect by post-treatment of vandetanib. HEK293 cells infected with SCoV2 at an MOI of 1 for $4 \mathrm{~h}$ 689 were washed with fresh cell culture media and then treated with vandetanib $(0.1,1$, or $10 \mu \mathrm{M})$ for 44 690 h. d, Anti-SCoV2 effect by pre-treatment of vandetanib. HEK293 cells treated with vandetanib (0.1, 6911 , or $10 \mu \mathrm{M}$ ) for $4 \mathrm{~h}$ were infected with SCoV2 at an MOI of 1 for $44 \mathrm{~h}$. Intracellular SCoV2 RNA 692 level in SCoV2-infected cells was analysed by qRT-PCR using PCR primers set specific to the SCoV2 $693 \mathrm{~N}$ gene (c-d). e, In vivo anti-SCoV2 efficacy of vandetanib in mouse model susceptible to SCoV2 694 infection. Wild-type aged mice (15 months old) intranasally inoculated with SCoV2 (1x10 $0^{5} \mathrm{pfu} / \mathrm{head}$, 695 clade S) were orally administrated with vandetanib daily. At 4 days post-infection, all mice was 696 terminated for further analysis. f, Intracellular SCoV2 RNA level in lung tissue isolated from SCoV2697 infected mice was analysed by qRT-PCR using PCR primers set specific to the SCoV2 N gene. g, 698 Additive anti-SCoV2 effect of vandetanib in combination with remdesivir. HEK293 cells infected with 
than the $\mathrm{IC}_{50}$ for $\left.\mathrm{SCoV} 2\right)$ in the presence or absence of vandetanib $(10 \mu \mathrm{M})$ for $48 \mathrm{~h}$. Intracellular

701 SCoV2 RNA level was analysed by qRT-PCR using PCR primers set specific to the SCoV2 N gene. P 702 values were calculated by one-way ANOVA (mean $\pm \mathrm{SD} ; \mathrm{n}=3 ; * \mathrm{P} \leq 0.0001)$.

704 Fig 5 | Vandetanib is a very effective against SCoV2 variants. a-b, Antiviral effect of vandetanib against SCoV2 variants. HEK293 cells infected with SARS-CoV-2 (S, V, G, GH, and GR clades) and its variants including United Kingdom variant (B.1.1.7) and South Africa variant (B.1.351) at an MOI of 1 , respectively, were treated with vandetanib for $48 \mathrm{~h}$. a, Total RNA isolated from cell culture media of SCoV2-infected cells was used for analysis of extracellular SCoV2 RNA level by qRT-PCR using PCR primers set specific to the SCoV2 N gene. b, Suppression of SCoV2 infectivity by vandetanib treatment was analysed by focus forming unit assay as described in the Materials and Methods.

712 Fig 6 | A schematic diagram showing a significant contribution of SCoV2-induced altered mitochondrial dynamics and mitochondrial EGFR accumulation in sustaining viral propagation.

714 First, SCoV2 RNA and nucleocapsid complex increases $\Delta \Psi \mathrm{m}$ during SCoV2 infection. This alteration

715 subsequently promotes mitochondrial elongation and swelling. SCoV2 also induces mitochondrial 716 biogenesis and activates mitochondrial OXPHOS process, thereby promoting abundant ATP 717 production. Second, SCoV2 activates EGFR-mediated cell survival signaling and subsequently 718 promotes mitochondrial EGFR internalization which contributes to maintenance of abnormal 719 mitochondrial bioenergetics. These alterations are physiologically relevant to the maintenance of 720 homoeostasis of SCoV2-infected cells and robust SCoV2 propagation 
723 Immunofluorescence microscopic analysis of SCoV2 infectivity in HEK293 cells. SCoV2-infected

724 cells were stained with SCoV2-N antibody (green) at 2 days post-infection. Nuclei, DAPI (blue);

725 Infection, SCoV2 nucleocapsid (N) antigen (green); accompanying graph, quantification of SCoV2-

726 N-positive cells. b, Real-time qRT-PCR data showing a rapid increase in intracellular SCoV2 RNA

727 level in HEK293 cells. Data, average of two independent experiments. c, Western blot data showing

728 the expression of spike and $\mathrm{N}$ antigens in SARS-CoV-2-infected HEK293 cells. Whole cell lysates of

729 SCoV2-infected cells were analysed by immunoblotting with antibodies specific to SCoV2 spike and

$730 \mathrm{~N}$ antigens. N, SCoV2 nucleocapsid; $\beta$-actin, an internal loading control.

732 Extended Data Fig. 2 | SCoV2 infection leads to the elevation in intracellular ATP synthesis and 733 prolonged survival of normal human primary bronchial cells. a, Confocal microscopy showing 734 SCoV2 infectivity in normal human primary bronchial cells. At 3 days post-infection, SCoV2-infected 735 cells were stained with SCoV2 spike antibody (red). Nuclei, DAPI (blue). b, Real-time qRT-PCR data 736 showing a rapid increase in intracellular SCoV2 RNA level in normal human primary bronchial cells. 737 Data, average of two independent experiments. c, Western blot data showing the expression of spike 738 and $\mathrm{N}$ antigens in SARS-CoV-2-infected normal human primary bronchial cells. Whole cell lysates of 739 SCoV2-infected cells were analysed by immunoblotting with antibodies specific to SCoV2 spike and 740 N antigens. N, SCoV2 nucleocapsid; $\beta$-actin, an internal loading control. d, Quantification of increased 741 intracellular ATP level in normal human primary bronchial cells infected with SCoV2 at an MOI of 1 742 at the indicated time points. Data, average of two independent experiments. e, Viability of SCoV2743 infected normal human primary bronchial cells. At 3 days post-infection, total number of normal 744 human primary bronchial cells infected with SCoV2 was measured using a hemocytometer and trypan 745 blue. f, Microscopic analysis of SCoV2-infected normal human primary bronchial cells at 5 days post746 infection. 
748 Extended Data Fig. 3 | SCoV2 does not induce cytopathic effect during robust virus propagation.

749 a, Light microscopic analysis of SCoV2-infected human-derived liver hepatoma Huh7 cells and lung 750 carcinoma epithelial A549 cells. At 3 days post-infection, cells were observed under a light microscope 751 at the BSL3 facility. b-c, Quantification of intracellular and extracellular SCoV2 RNA levels in Huh7 752 and A549 cells. Cells were infected with SCoV2 at an MOI of 1 for 3 days. Total RNAs purified from 753 SCoV2-infected cells or culture supernatants were used for analyses of intracellular and extracellular 754 SCoV2 RNA levels by real-time qRT-PCR using PCR primers set specific to the SCoV2 N gene. Data, 755 average of two independent experiments.

757 Extended Data Fig. 4 | Wild-type aged mouse susceptible to SCoV2 infection. a, Wild-type 758 C57BL/6 aged mice (15 months old) were intranasally inoculated with SCoV2 (1x10 5 pfu/head, clade 759 S). b-c, Body weight and survival rate of SCoV2-infected mice were monitored daily. d, At 1, 3, and 7607 days post-infection, uninfected and SCoV-2-infected mice was terminated for further analysis. 761 Intracellular SCoV2 RNA level in lung tissue isolated from uninfected and SCoV2-infected mice was 762 analysed by qRT-PCR using PCR primers set specific to the SCoV2 N gene. 
Table 1. SARS-CoV-2 viruses used in this study

\begin{tabular}{|c|c|c|c|c|c|}
\hline Virus & Clade & & $\begin{array}{l}\text { NCCP* } \\
\text { number }\end{array}$ & $\begin{array}{c}\text { Cell line for virus } \\
\text { propagation }\end{array}$ & $\begin{array}{c}\text { Virus stock } \\
\text { concentration }^{* *}\end{array}$ \\
\hline \multirow{7}{*}{ SARS-CoV-2 } & $\mathrm{S}$ & & 43326 & \multirow{7}{*}{ Vero E6 } & $1 \times 10^{6} \mathrm{pfu} / \mathrm{ml}$ \\
\hline & $\mathrm{V}$ & & 43342 & & $5 \times 10^{5} \mathrm{pfu} / \mathrm{ml}$ \\
\hline & $\mathrm{G}$ & & 43343 & & $1 \times 10^{6} \mathrm{pfu} / \mathrm{ml}$ \\
\hline & GR & & 43344 & & $1 \times 10^{6} \mathrm{pfu} / \mathrm{ml}$ \\
\hline & $\mathrm{GH}$ & & 43345 & & $1 \times 10^{6} \mathrm{pfu} / \mathrm{ml}$ \\
\hline & GR & $\begin{array}{l}\text { UK variant } \\
\text { (B.1.1.7) }\end{array}$ & 43381 & & $1 \times 10^{7} \mathrm{pfu} / \mathrm{ml}$ \\
\hline & $\mathrm{GH}$ & $\begin{array}{l}\text { SA Variant } \\
\text { (B.1.351) }\end{array}$ & 43382 & & $1 \times 10^{7} \mathrm{pfu} / \mathrm{ml}$ \\
\hline
\end{tabular}

766 *NCCP: National Culture Collection for Pathogens, National Institute of Health, South Korea

$767^{* *}$ After virus propagation using Vero cell, virus concentration was titrated by plaque assay 
Table 2. Summary of FDA-approved EGFR inhibitors showing anti-SARS-CoV-2 efficacy

\begin{tabular}{|c|c|c|c|c|}
\hline $\begin{array}{c}\text { EGFR } \\
\text { inhibitors } \\
\text { (TKIs) }\end{array}$ & Structure & $\begin{array}{c}\text { Final } \\
\text { concentration } \\
\text { for primary } \\
\text { screening }\end{array}$ & Cytotoxicity* & $\begin{array}{c}\text { Anti-SARS- } \\
\text { CoV-2 } \\
\text { efficacy }^{* *}\end{array}$ \\
\hline Gefitinib & & $10 \mu \mathrm{M}$ & $\begin{array}{c}- \\
\text { (negative) }\end{array}$ & $\begin{array}{c}\geq 4.1 \times 10^{1} \\
\text { RNA }^{* * *}\end{array}$ \\
\hline Olmutinib & & $10 \mu \mathrm{M}$ & $\begin{array}{c}- \\
\text { (negative) }\end{array}$ & $\begin{array}{c}\geq 7.6 \times 10^{3} \\
\text { RNA }^{* * *}\end{array}$ \\
\hline Erlotinib & & $10 \mu \mathrm{M}$ & $\begin{array}{c}- \\
\text { (negative) }\end{array}$ & $\begin{array}{c}\geq 1.6 \times 10^{3} \\
\text { RNA }^{* * * *}\end{array}$ \\
\hline Lapatinib & & $10 \mu \mathrm{M}$ & $\begin{array}{c}- \\
\text { (negative) }\end{array}$ & $\begin{array}{c}\geq 2.4 \times 10^{3} \\
\text { RNA }^{* * *}\end{array}$ \\
\hline Bosutinib & & $10 \mu \mathrm{M}$ & $\begin{array}{c}- \\
\text { (negative) }\end{array}$ & $\begin{array}{c}\geq 1.4 \times 10^{3} \\
\text { RNA }^{* * *}\end{array}$ \\
\hline Cabozantinib & & $10 \mu \mathrm{M}$ & $\begin{array}{c}- \\
\text { (negative) }\end{array}$ & $\begin{array}{c}\geq 1.4 \times 10^{4} \\
\text { RNA }^{* * *}\end{array}$ \\
\hline Icotinib & & $10 \mu \mathrm{M}$ & $\begin{array}{c}- \\
\text { (negative) }\end{array}$ & $\begin{array}{c}\geq 4.0 \times 10^{3} \\
\text { RNA }^{* * *}\end{array}$ \\
\hline Vandetanib & & $10 \mu \mathrm{M}$ & $\begin{array}{c}- \\
\text { (negative) }\end{array}$ & $\begin{array}{c}\geq 1.9 \times 10^{4} \\
\text { RNA }^{* * * *}\end{array}$ \\
\hline
\end{tabular}




\begin{tabular}{l|l|l|l|l}
\hline Brigatinib & $10 \mu \mathrm{M}$ & $\begin{array}{c}- \\
\text { (negative) }\end{array}$ & $\begin{array}{c}\geq 4.1 \times 10^{2} \\
\mathrm{RNA}^{* * *}\end{array}$ \\
\hline Nacomitinib & $10 \mu \mathrm{M}$ & $\begin{array}{c}+ \\
\text { (positive) }\end{array}$ & N.D.*** \\
\hline (positive) & N.D.*** \\
\hline
\end{tabular}

771 *In the presence of EGFR inhibitors for $48 \mathrm{~h}$, cytotoxicity of SARS-CoV-2-infected HEK293 cells was 772 observed by light microscope in biosafety laboratory 3 facility.

$773{ }^{* *}$ Anti-SARS-CoV-2 efficacy of EGFR inhibitors was determined by extracellular SARS-CoV-2 RNA

774 level analysed by real-time quantitative RT-PCR with primer set specific to SARS-CoV-2 N gene.

$775^{* * *}$ Unit: copies/ $\mu 1$

${ }^{* * * * *}$ N.D.: Not determined 
Fig. 1

a

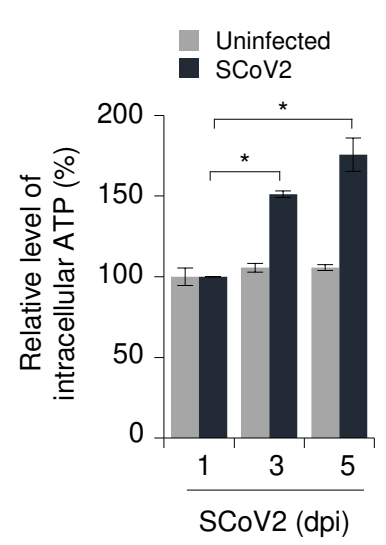

b
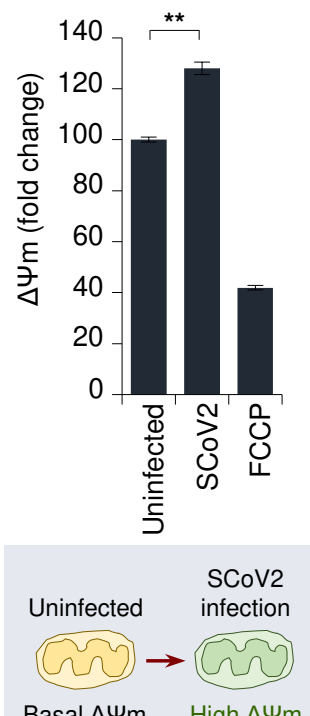

d

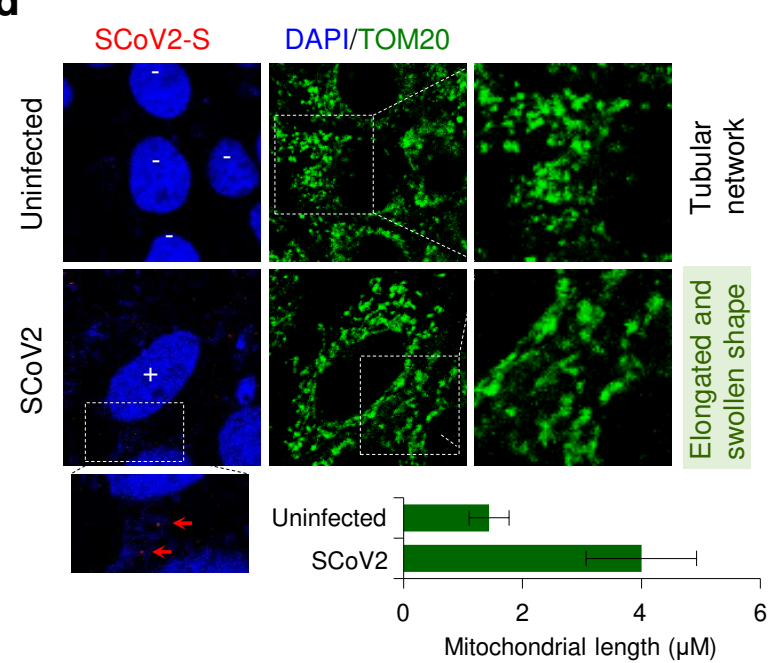

Shin et al.
C

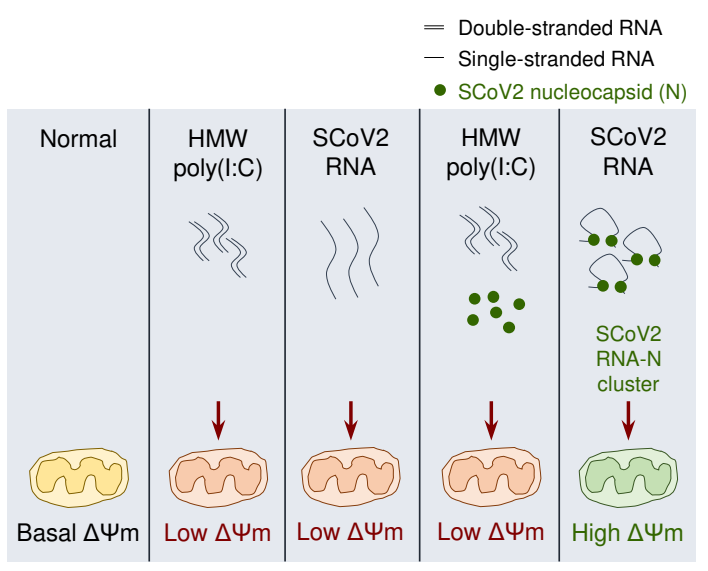

e

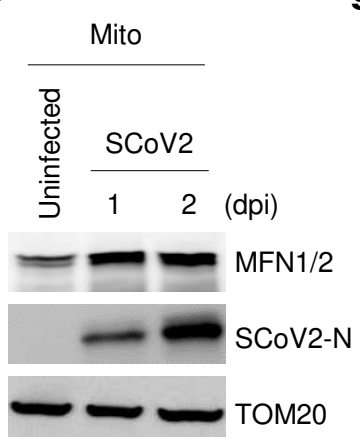

f

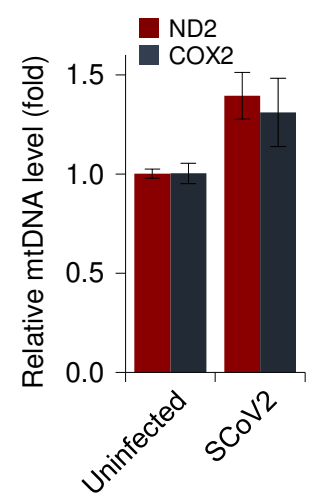

g

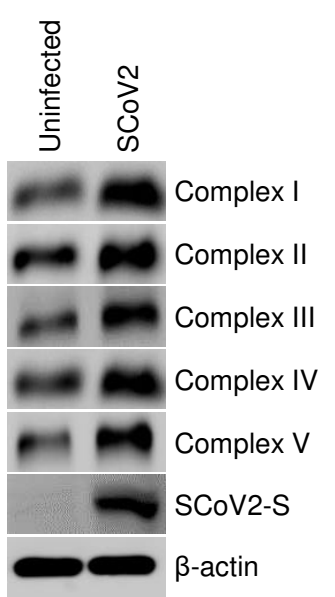

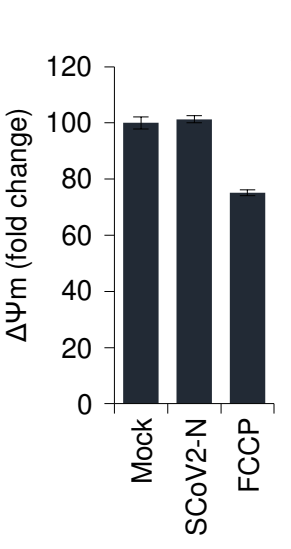

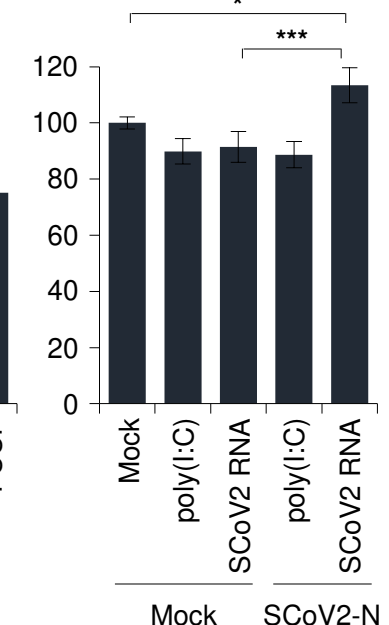

h

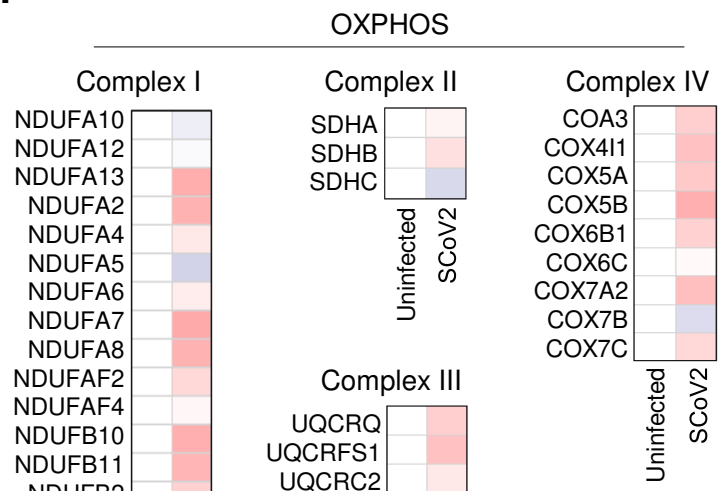

Complex V

ATP5F1A ATP5F1B ATP5F1C ATP5F1D ATP5IF1
ATP5ME ATP5PB ATP5PO DMAC2L

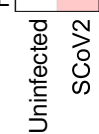


Fig. 2

a

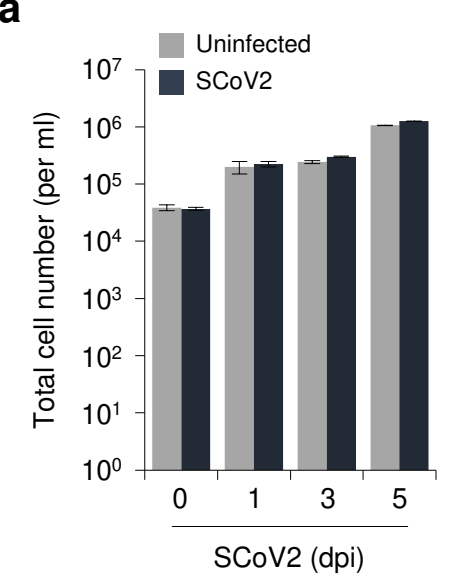

e

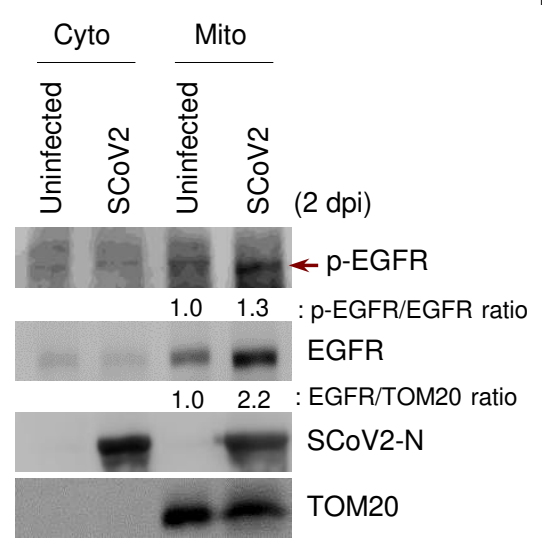

b

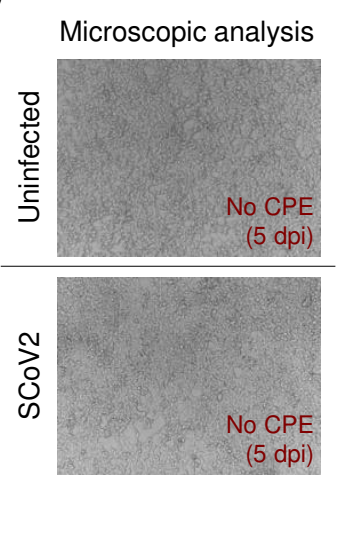

f

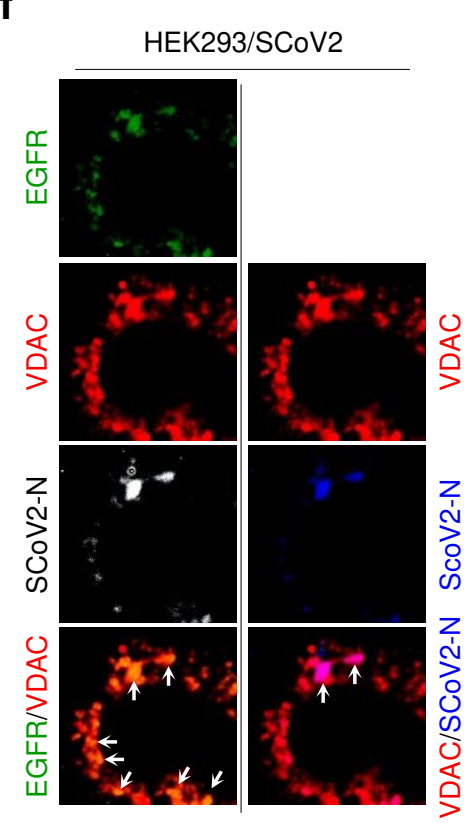

d
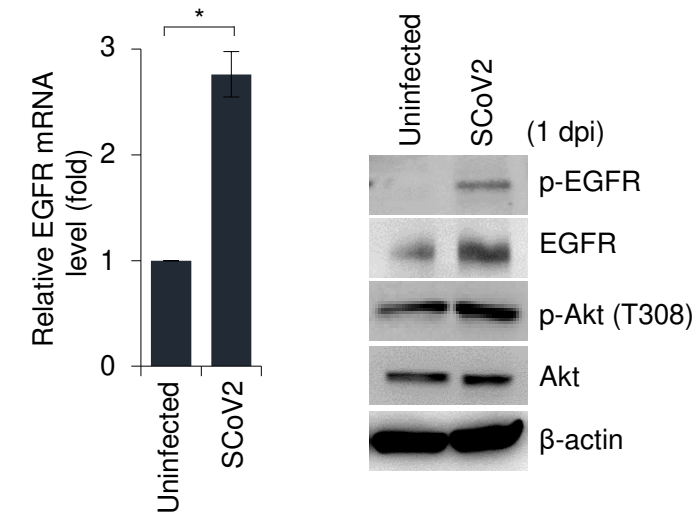

g Wild-type aged mice (15 months old) SCoV2 (IN)

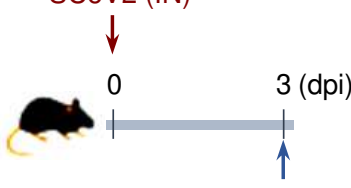

Lung tissue isolation Mito $\quad$ Mitochondria isolation

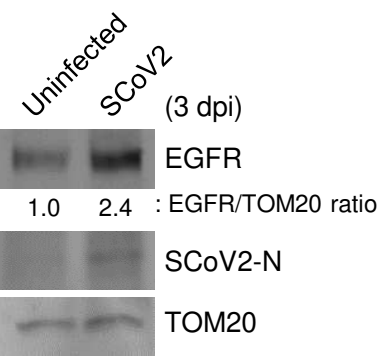


Fig. 3

a

Experimental scheme for analysis of anti-SCoV2 efficacy by EGFR inhibitors

HEK293 cell culture

$$
\begin{gathered}
\text { SCoV2 infection } \\
(1 \mathrm{MOI} ; 4 \mathrm{~h})
\end{gathered}
$$

$\downarrow$

Washing of SCoV2-infected cells \& incubation with EGFR inhibitor (44 h)
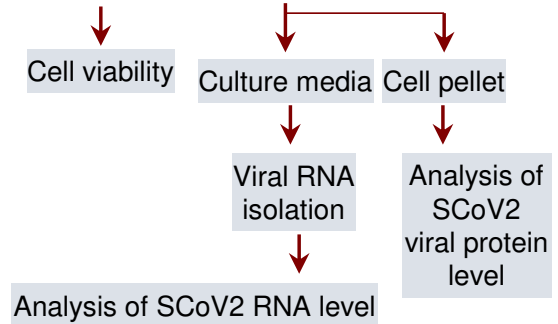

d

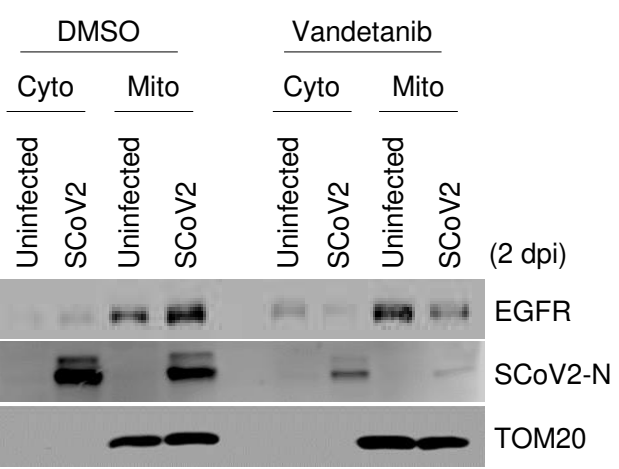

b

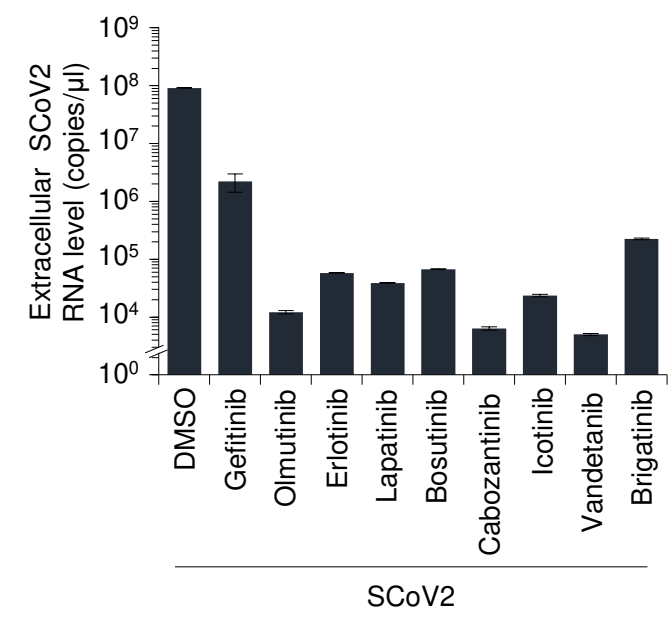

e

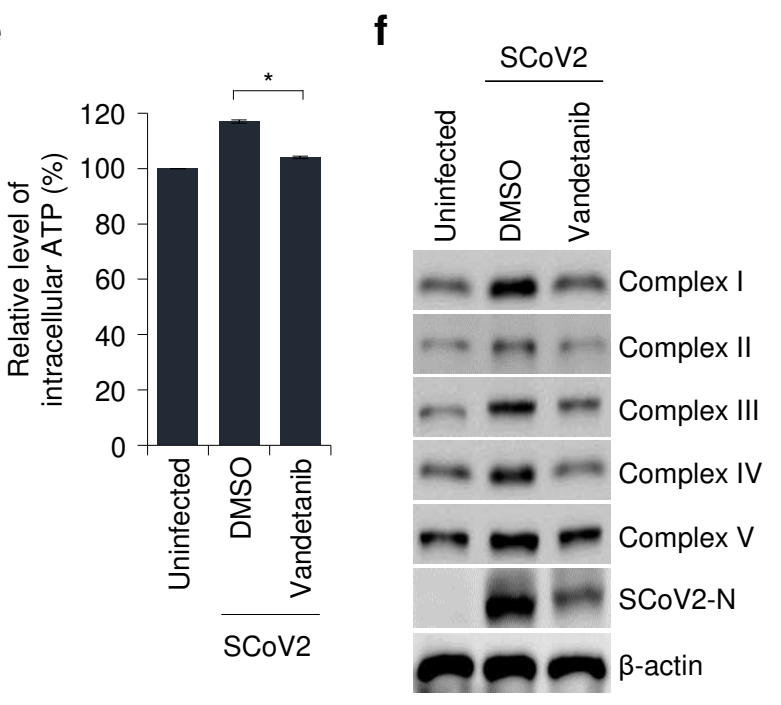

C

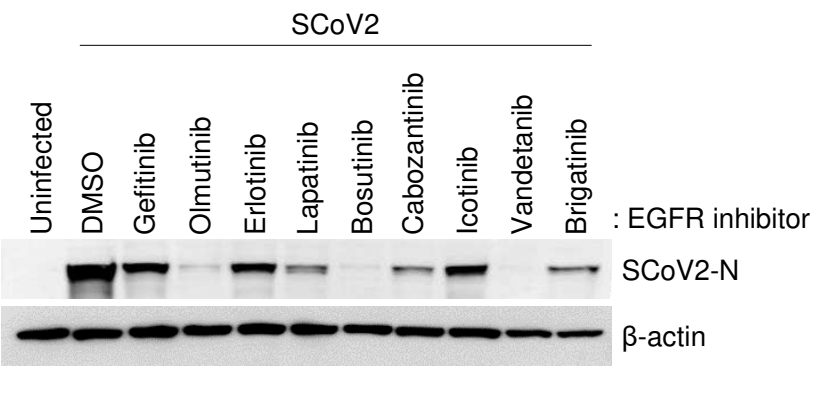

\begin{tabular}{|c|c|c|c|}
\hline \multicolumn{4}{|c|}{ OXPHOS } \\
\hline Complex I & Complex II & \multicolumn{2}{|c|}{ Complex IV } \\
\hline NDUFA10 & SDHA & COA3 & \\
\hline NDUFA12 & SDHB & COX4I1 & \\
\hline NDUFA13 & SDHC & COX5A & \\
\hline NDUFA2 & & COX5B & \\
\hline NDUFA4 & Comn & COX6B1 & \\
\hline NDUFA5 & & COX6C & \\
\hline NDUFA6 & UQCRQ & COX7A2 & \\
\hline NDUFA7 & UQCRFS1 & CoX7B & \\
\hline NDUFA8 & UQCRC2 & COX7C & \\
\hline
\end{tabular}

g

NDUFAF2

NDUFAF4

NDUFB10

NDUFB11

NDUFB2

NDUFB3

NDUFB4

NDUFB5

NDUFB6

NDUFB7

NDUFB8

NDUFB9

NDUFC2

NDUFS1

NDUFS2

NDUFS3
NDUFS7

NDUFS7

NDUFV

UQCRC1

UQCRB

UQCR11

COQ9

Complex V

ATP5F1A ATP5F1B

ATP5F1C

ATP5F1C

ATP5IF1

ATP5ME

ATP5PB

ATP5PO

ATPSPO

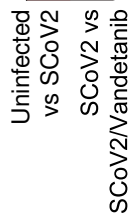


Fig. 4

a

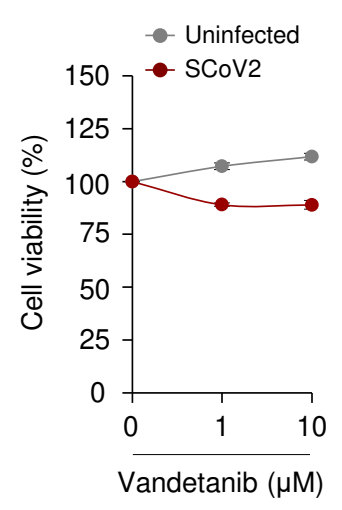

e

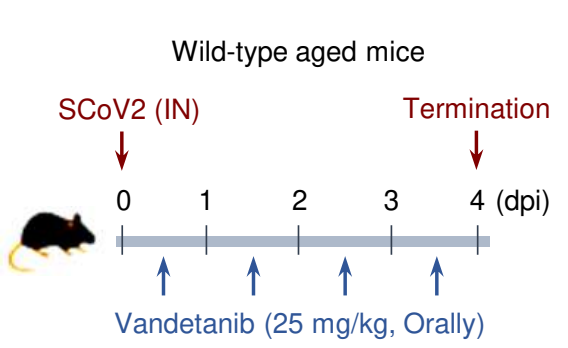

b

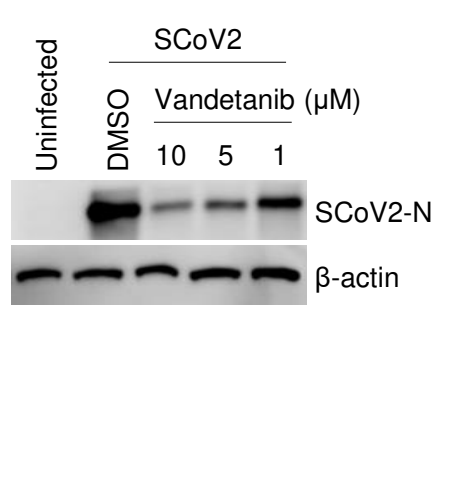

C

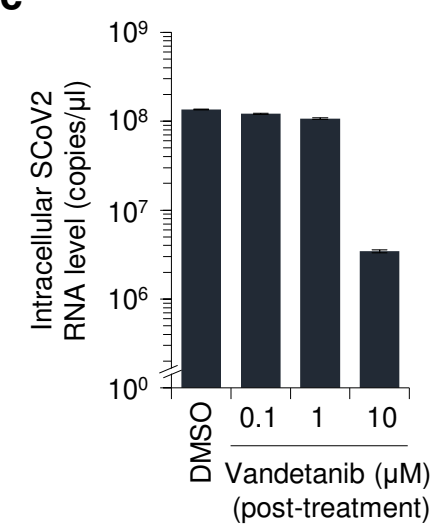

d
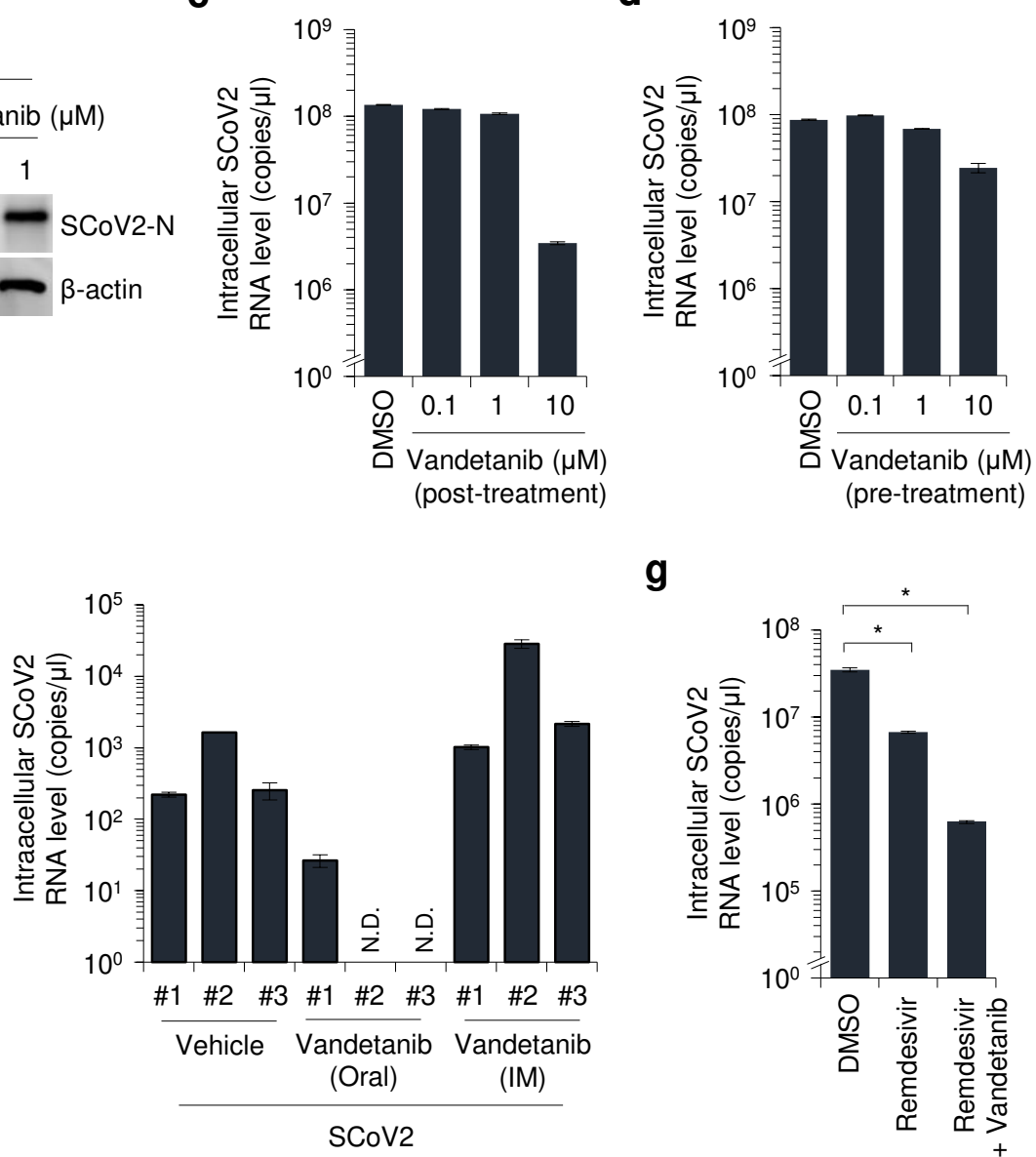

g

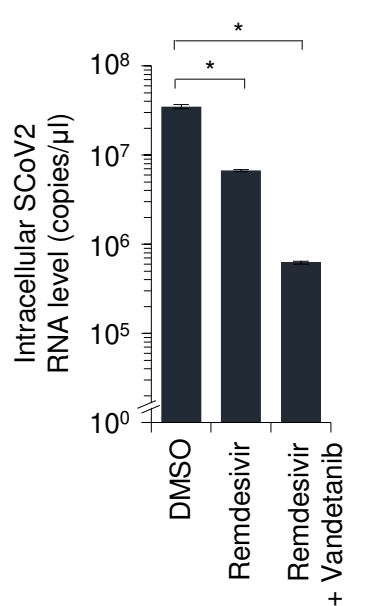




\section{Fig. 5}

a
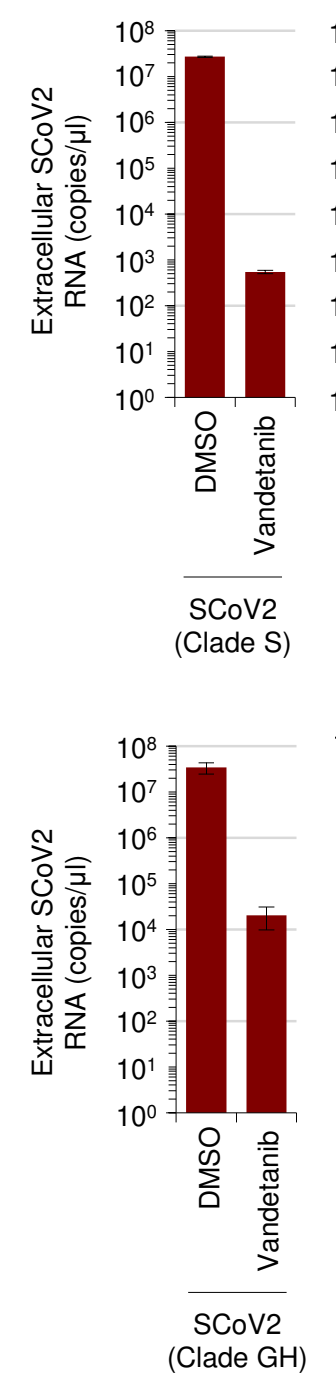
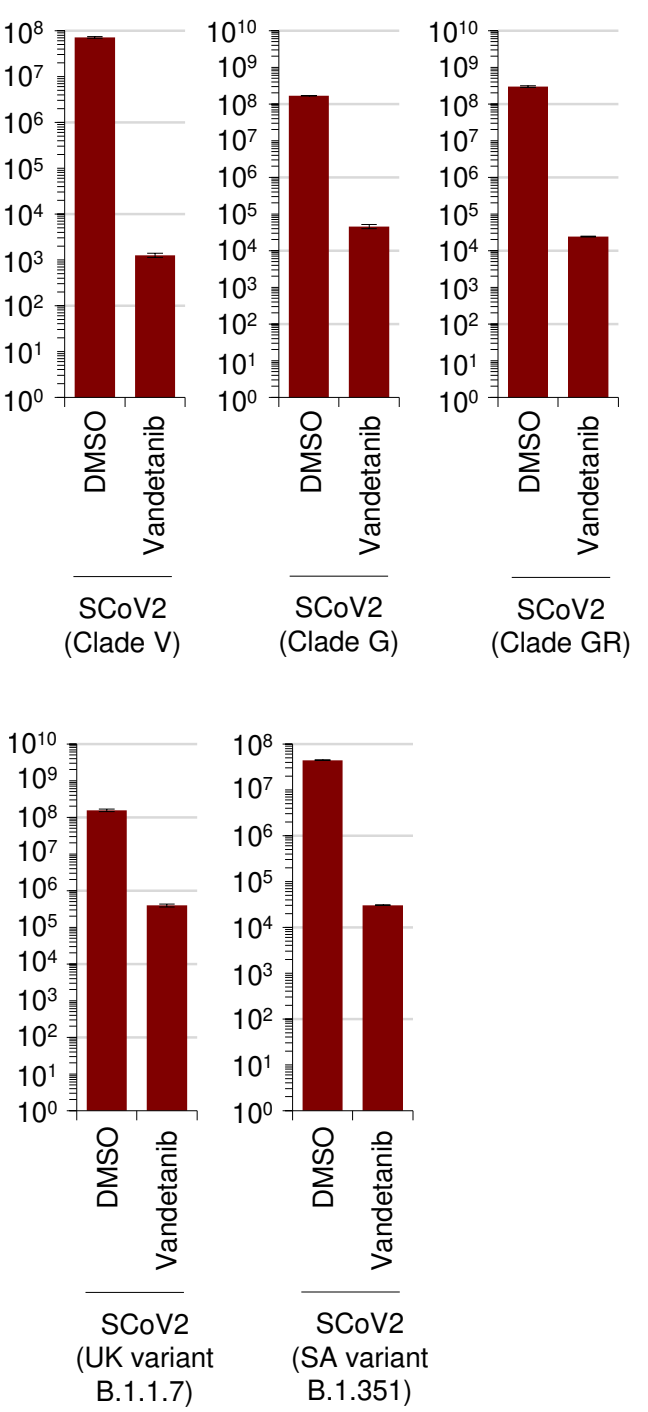

b

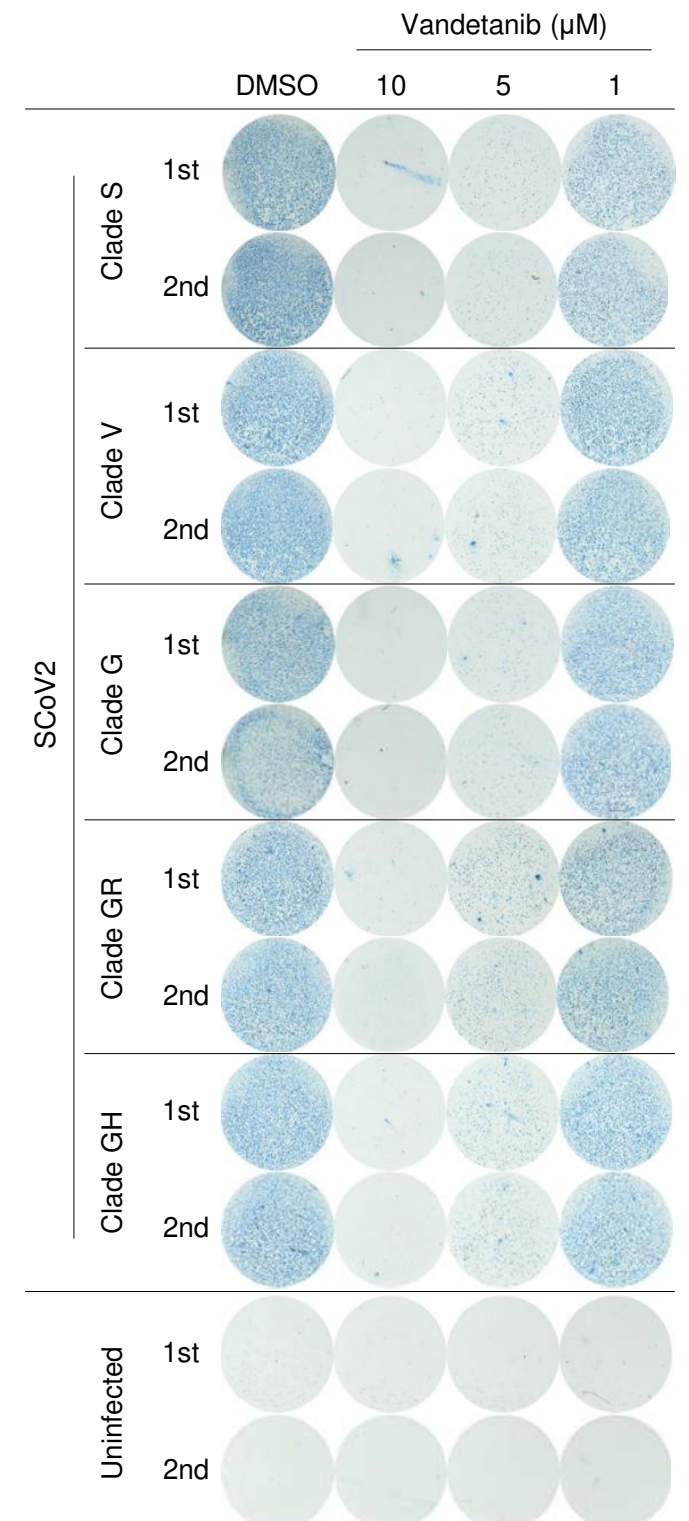

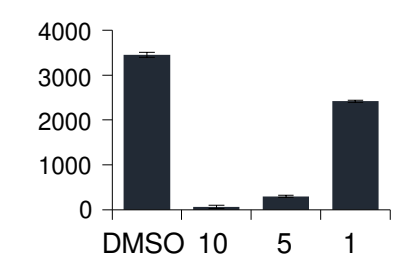
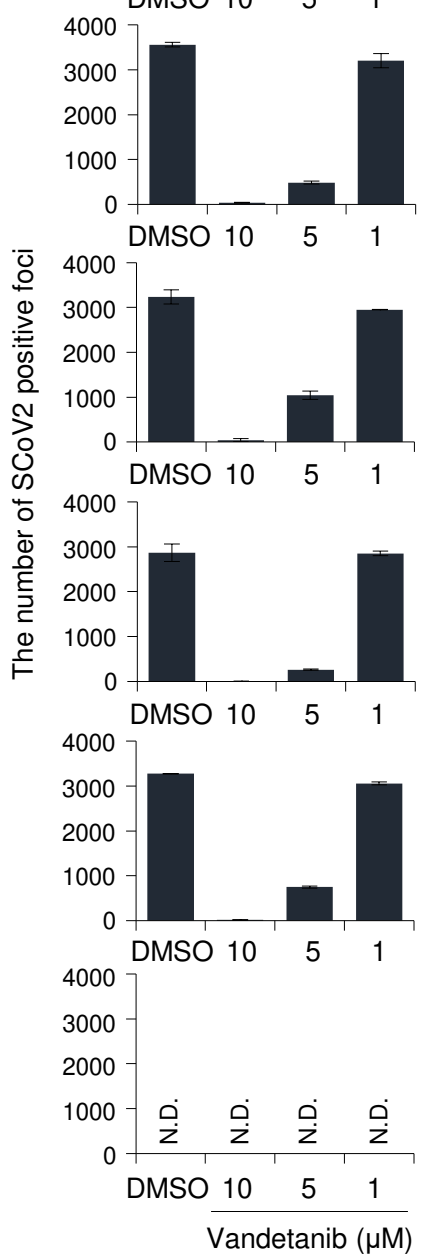
Fig. 6

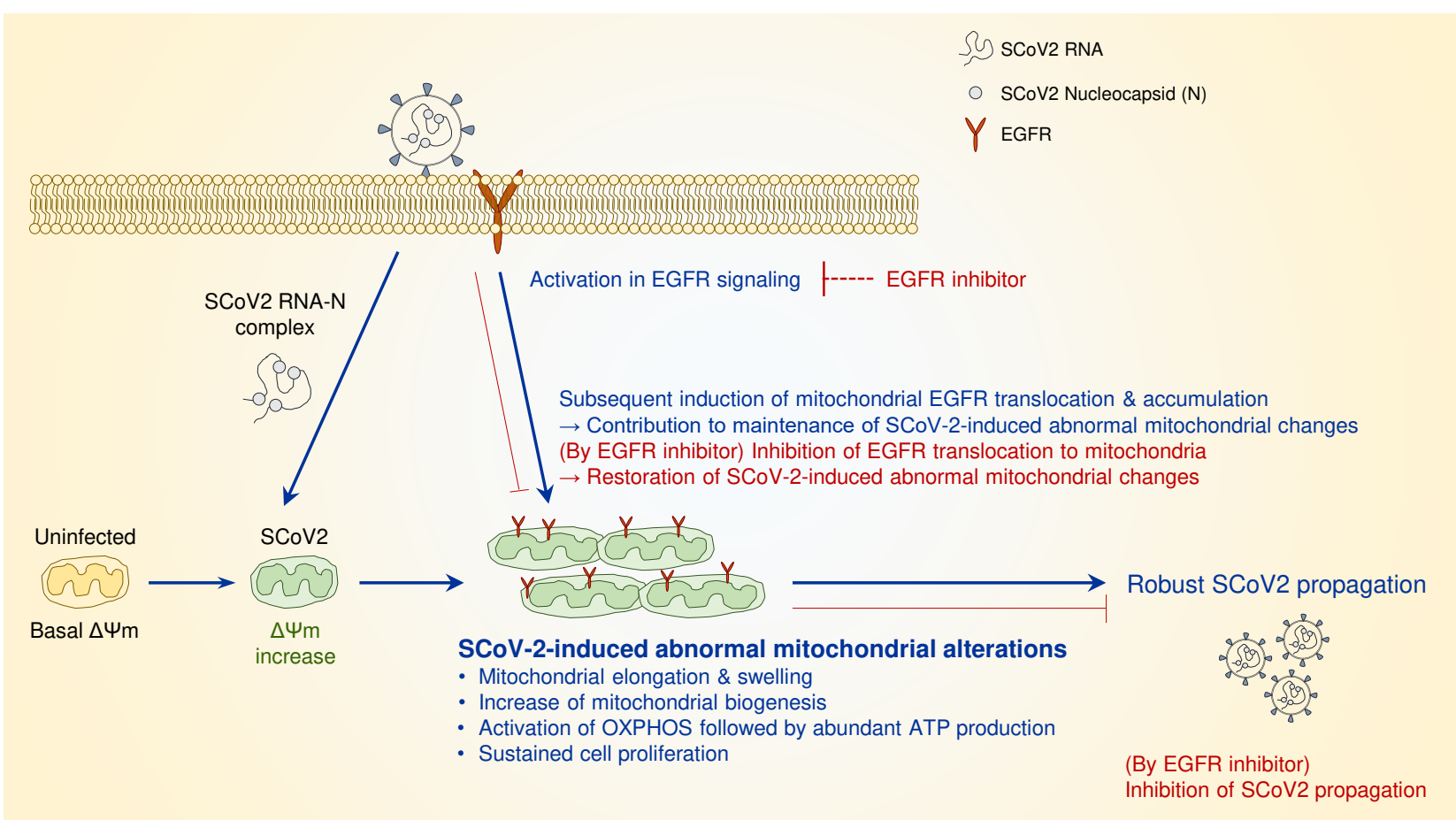




\section{Extended Data Fig. 1}

a

\section{- DAPI}

Uninfected SCoV2 (2 dpi)

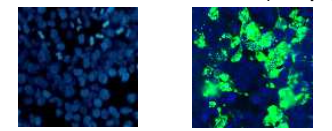

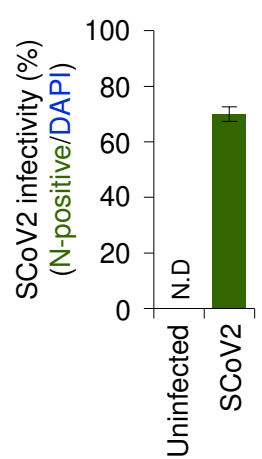

b

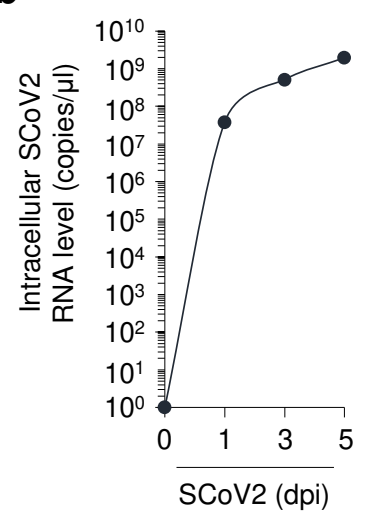

C

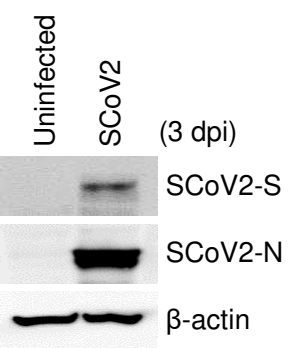




\section{Extended Data Fig. 2}

a

Normal human primary bronchial cells

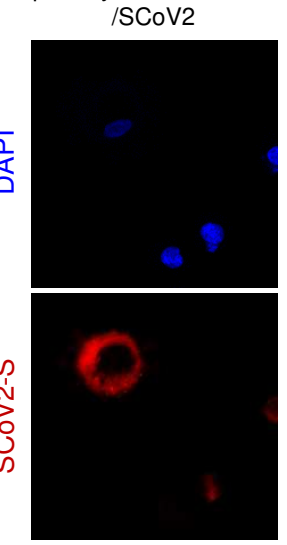

d
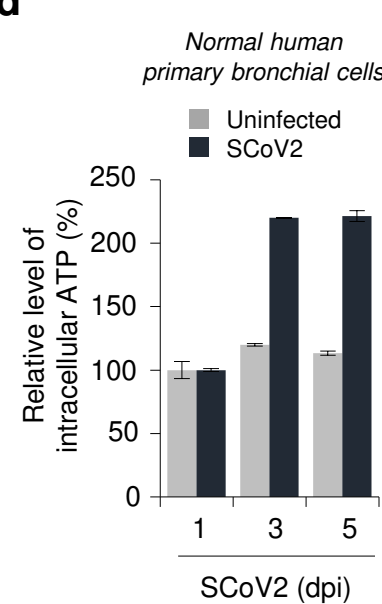

b
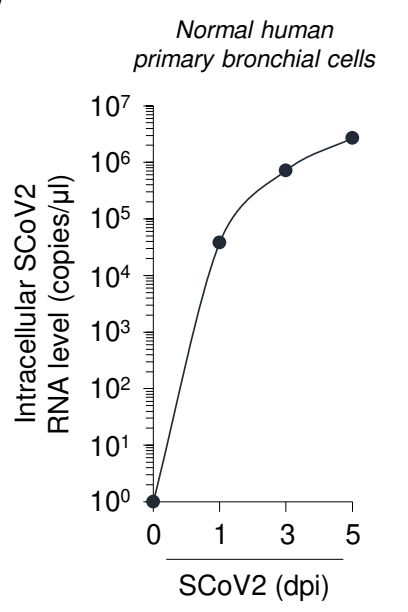

e

$$
\begin{gathered}
\text { Normal human } \\
\text { primary bronchial cells }
\end{gathered}
$$

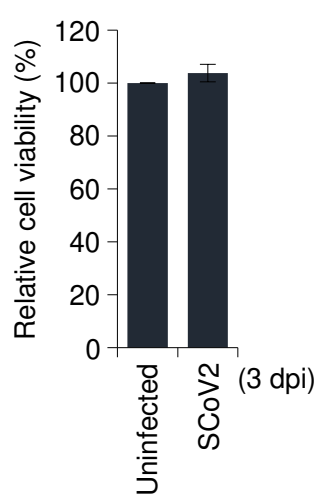

C

Normal human primary bronchial cells

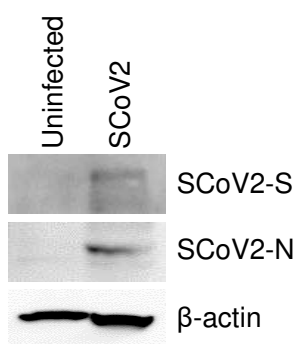

Microscopic analysis

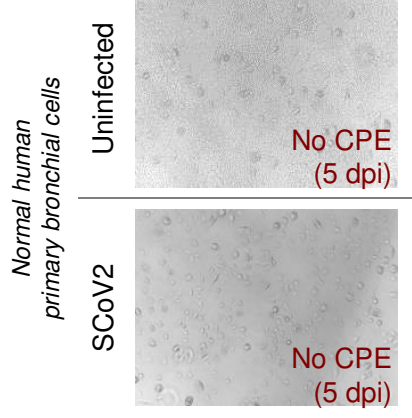




\section{Extended Data Fig. 3}

a

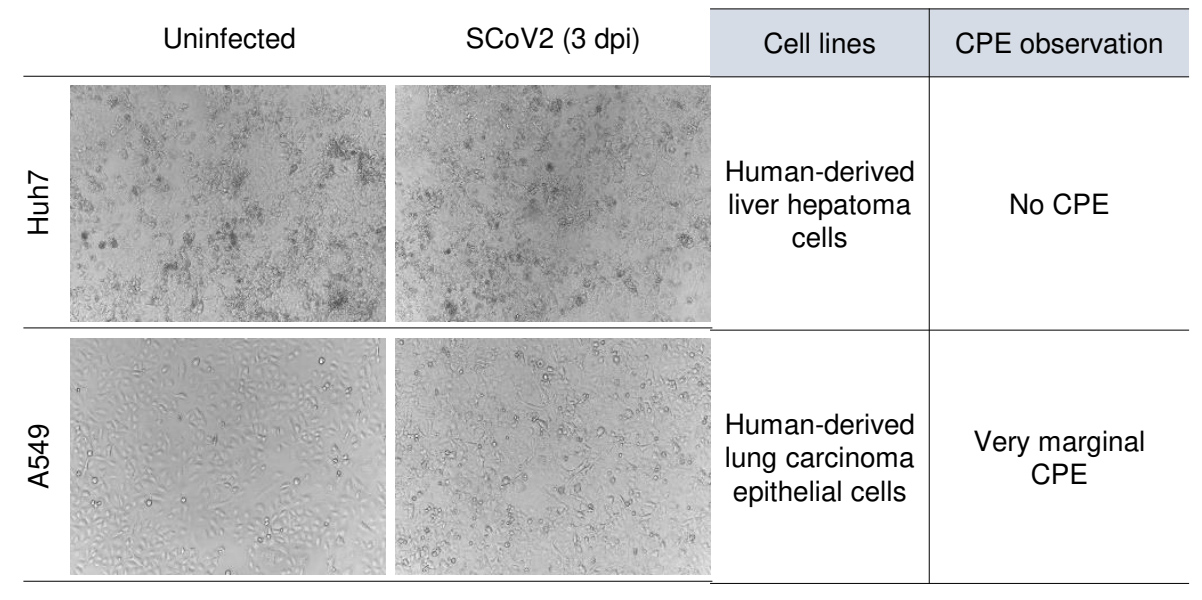

b

C

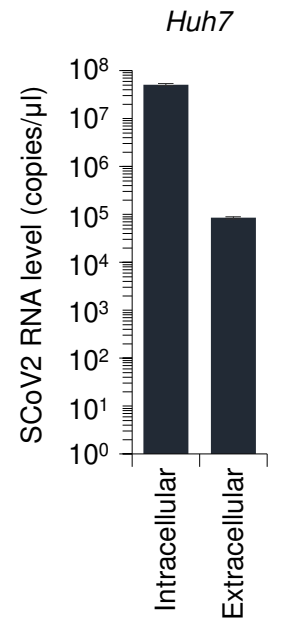

A549

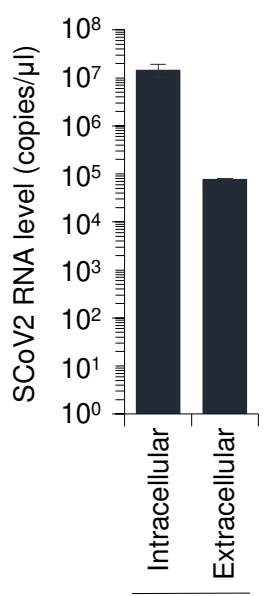

SCoV2 (3 dpi)

SCoV2 (3 dpi) 


\section{Extended Data Fig. 4}

a

Wild-type aged mice

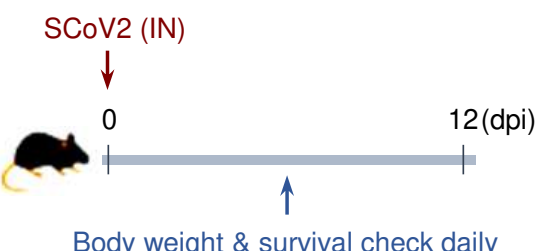

Body weight \& survival check daily

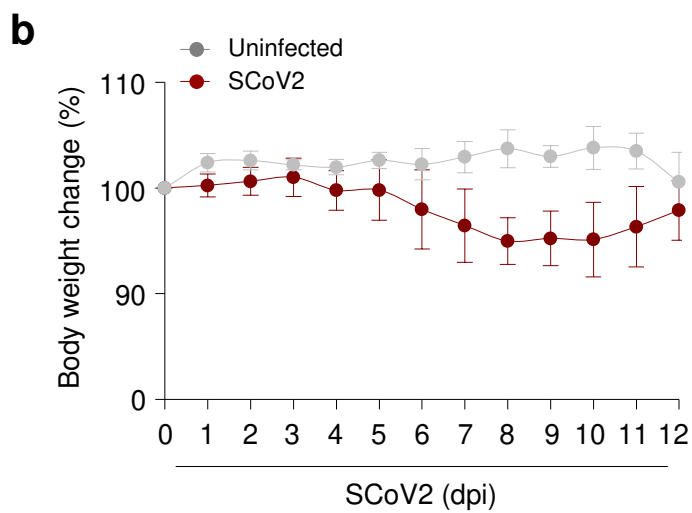

C
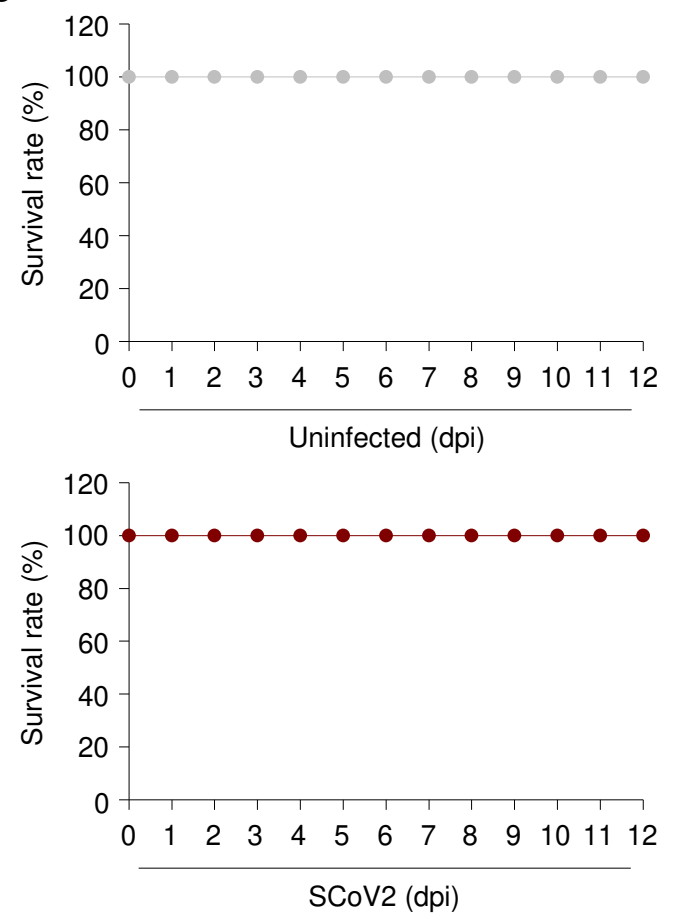

d

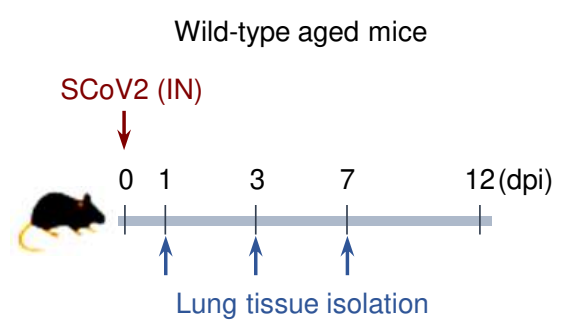

Wild-type aged mice lung tissue

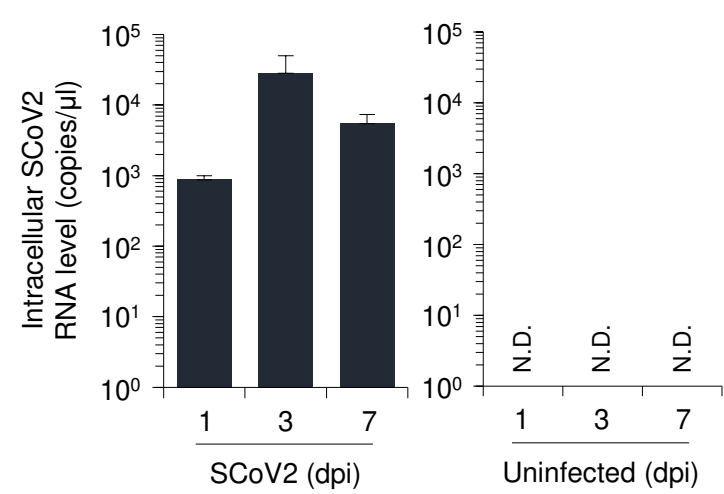

